\title{
Predictive models for kidney disease: improving global outcomes (KDIGO) defined acute kidney injury in UK cardiac surgery
}

Kate Birnie ${ }^{1}$, Veerle Verheyden ${ }^{2}$, Domenico Pagano ${ }^{3}$, Moninder Bhabra $^{4}$, Kate Tilling ${ }^{1}$, Jonathan A Sterne ${ }^{1}$, Gavin J Murphy ${ }^{2^{*}}$ and on behalf of the UK AKI in Cardiac Surgery Collaborators

\begin{abstract}
Introduction: Acute kidney injury (AKI) risk prediction scores are an objective and transparent means to enable cohort enrichment in clinical trials or to risk stratify patients preoperatively. Existing scores are limited in that they have been designed to predict only severe, or non-consensus AKI definitions and not less severe stages of AKI, which also have prognostic significance. The aim of this study was to develop and validate novel risk scores that could identify all patients at risk of AKI.
\end{abstract}

Methods: Prospective routinely collected clinical data $(n=30,854)$ were obtained from 3 UK cardiac surgical centres (Bristol, Birmingham and Wolverhampton). AKI was defined as per the Kidney Disease: Improving Global Outcomes (KDIGO) Guidelines. The model was developed using the Bristol and Birmingham datasets, and externally validated using the Wolverhampton data. Model discrimination was estimated using the area under the ROC curve (AUC). Model calibration was assessed using the Hosmer-Lemeshow test and calibration plots. Diagnostic utility was also compared to existing scores.

Results: The risk prediction score for any stage AKI (AUC $=0.74$ ( $95 \%$ confidence intervals $(C I)$ 0.72, 0.76)) demonstrated better discrimination compared to the Euroscore and the Cleveland Clinic Score, and equivalent discrimination to the Mehta and Ng scores. The any stage AKI score demonstrated better calibration than the four comparison scores. A stage $3 \mathrm{AKI}$ risk prediction score also demonstrated good discrimination ( $\mathrm{AUC}=0.78(95 \% \mathrm{Cl} 0.75,0.80)$ ) as did the four comparison risk scores, but stage $3 \mathrm{AKI}$ scores were less well calibrated.

Conclusions: This is the first risk score that accurately identifies patients at risk of any stage AKI. This score will be useful in the perioperative management of high risk patients as well as in clinical trial design.

\section{Introduction}

Acute kidney injury (AKI) is a common and severe complication of cardiac surgery affecting up to $30 \%$ of all patients and increasing mortality up to fourfold $[1,2]$. No effective treatment has been identified despite over 70 randomised controlled trials (RCTs) of proposed renoprotective interventions [3,4]. Systematic reviews have documented important limitations in these trials such as the enrolment of low or mixed AKI-risk patient cohorts, small sample sizes, and low statistical power $[3,4]$.

\footnotetext{
*Correspondence: gjm19@le.ac.uk

${ }^{2}$ Department of Cardiovascular Sciences, University of Leicester, Leicester NIHR Cardiovascular Biomedical Research Unit, Clinical Sciences Wing, Glenfield General Hospital, Leicester LE3 9QP, UK

Full list of author information is available at the end of the article
}

These are important sources of bias that will have increased the likelihood of negative trial results. Recent recommendations on the design of trials in AKI suggest that these limitations may be countered by cohort enrichment [5], whereby the enrolment of patients with higher event rates will permit targeting of interventions to those patients populations most likely to benefit, smaller sample sizes, and increased study power. AKI risk prediction scores are an objective, transparent means of cohort enrichment but are not widely used. This is because existing scores have been developed principally to identify patients at risk of renal replacement therapy (RRT) which is rare and not less severe AKI, which is also associated with poor prognosis but occurs with greater frequency [6]. There is also uncertainty as to 
the generalizability of these scores. Only two published scores, The Cleveland clinic score [7], and the Mehta score [8] have been independently validated, and neither has demonstrated adequate discrimination and calibration in non-North American patient populations $[9,10]$.

The aim of this study was to develop and externally validate two novel risk scores that could be used for cohort enrichment in clinical trials of renoprotective interventions in cardiac surgery. We used the Kidney Disease: Improving Global Outcomes (KDIGO) guidelines AKI definition [11] as this reconciles important differences between the two earlier consensus definitions; Acute Kidney Injury Network (AKIN) [12] and risk, injury, failure, loss, end stage (RIFLE) [13]. The prognostic utility of this score has only been demonstrated thus far in a single centre [14]. To support the validity and utility of the risk scores our first objective was therefore to demonstrate the utility of the KDIGO definition in a large multicentre cohort. Next, we developed two new scores based on preoperative variables; one to identify all patients at risk of AKI (KDIGO stages 1 to 3 ), and the second to identify only those patients at risk of severe AKI (KDIGO stage 3). Finally we compared the diagnostic utility of these new scores with four published risk scores.

\section{Methods}

This study was approved by the South West research ethics committee under reference 11/SW/0075. The requirement for written informed consent was waived. Our research objectives and methods were specified prior to execution.

\section{Study population}

This retrospective cohort study used routinely collected data from the patient analysis and tracking system (PATS), from three UK hospitals. We obtained prospectively collected data on all adult cardiac patients for the following periods: 1996 to 2010 at University Hospitals of Bristol, 2002 to 2010 at University Hospitals of Birmingham National Health Service (NHS) Foundation Trust, and 2004 to 2010 at Wolverhampton Heart and Lung Centre. Our analyses included all patients aged $\geq 16$ years, who underwent cardiac surgery, with or without cardiopulmonary bypass (CBP), and including those who underwent surgery to the thoracic aorta; we excluded patients already on renal replacement therapy (RRT) or who had received a kidney transplants, and patients who died in theatre.

Definitions of perioperative variables used were consistent across the three sites, and are as specified by the UK National Adult Cardiac Surgery Audit (NACSA) [15]. NACSA is a component of the UK cardiac surgery national quality assurance programme, whereby a defined set of perioperative data are collected prospectively by the anaesthetist, surgeon, and ICU, high-dependency unit (HDU), and ward nurses on the PATS databases. These data are submitted to the National Institute Clinical Outcome Research (NICOR) for analysis and the generation of risk-adjusted outcome data [16]. The data undergo routine internal quality assurance prior to submission as well as external data quality assessment by NICOR. The NACSA Annual Report for 2010 indicated that these three centres are among the best units nationally for data quality [17]. PATS data were linked using name, hospital number, and date of birth, to institutional biochemistry databases that record serial serum creatinine results, to identify those patients who developed AKI. During the study serum creatinine was measured using the Jaffe method at all three centres. Wolverhampton and Birmingham used the Roche Modular system (Roche Diagnostics, Ltd, Lewes, UK). Bristol used the Olympus Diagnostics System AU640 or AU2700 (Olympus Diagnostic Systems, Southall, UK). The assays were calibrated using the manufacturers' controls with inter-institutional diagnostic accuracy monitored by a national (UK) quality assessment scheme.

\section{Definitions}

\section{Measurement of acute kidney injury}

AKI was classified according to the KDIGO guidelines [11]. Stage-1 AKI was defined as an increase from baseline of $\geq 26 \mu \mathrm{mol} / \mathrm{L}$ of postoperative creatinine or an increase of 1.5 to 1.9 times the preoperative creatinine within 7 days; stage 2 was an increase of 2.0 to 2.9 times the preoperative creatinine; stage- 3 AKI was an increase $\geq 3$ times the preoperative creatinine or an increase to $\geq 354 \mu \mathrm{mol} / \mathrm{L}$ or when the patient commenced RRT. The RRT was administered for uraemia, volume overload, or biochemical abnormalities, according to institutional protocols. Urine output data were not available and therefore not used in our AKI definition. The baseline creatinine value was defined as the preoperative value obtained closest to the date of the operation. Cases where the baseline data were missing, most commonly emergency and salvage patients, were not included in the complete case analyses.

\section{Demographic factors}

Information was extracted from PATS on age (grouped into three categories: $<60$; 60 to 74 ; or $\geq 75$ years), sex, body mass index (BMI) (grouped into five categories: <20.0; 20.0 to $24.9 ; 25.0$ to $29.9 ; 30.0$ to 34.9 ; or $\geq 35.0 \mathrm{~kg} / \mathrm{m}^{2}$ ) and smoking status (never smoked; ex-smoker; or current smoker).

\section{Preoperative factors}

Information on prespecified factors was obtained from PATS for the three centres. The presence of angina was 
grouped according to the Canadian Cardiovascular Society (CCS) categories: no angina, CCS 1 (ordinary activity does not cause angina); CCS 2 (slight limitation); CCS 3 (moderate limitation); or CCS 4 (inability to carry out any physical activity without discomfort). Dyspnoea was grouped according to the New York Heart Association (NYHA) functional classification: NYHA 1 (no symptoms and no limitation in ordinary physical activity); NHYA 2 (mild symptoms and slight limitation during ordinary activity); NYHA 3 (marked limitation in activity due to symptoms, comfortable only at rest); or NYHA 4 (severe limitations, experiences symptoms even while at rest). Previous myocardial infarction (MI) was categorised as; none; 1 ; or $\geq 2$. Information was obtained on previous cardiac, vascular or thoracic surgery $(0$ and $\geq 1)$, the presence of diabetes, peripheral vascular disease, pulmonary disease, neurological disease, hypertension (treated or blood pressure (BP) $>140 / 90$ ) and preoperative haemoglobin $(<10.0 ; 10.0$ to 11.9 ; or $\geq 12.0 \mathrm{~g} / \mathrm{dL})$. Glomerular filtration rate (GFR) was estimated from the Cockcroft-Gault equation using the preoperative creatinine value obtained closest to the day of surgery, and grouped as: $<30.0 ; 30.0$ to $59.9 ; 60.0$ to 89.9 ; or $\geq 90 \mu \mathrm{mol} / \mathrm{L}$. Heparin or nitrates usage was grouped according to: none; within a week; or at operation. Any critical preoperative event was considered to be cardiogenic shock; preoperative intravenouse (IV) inotropes; or preoperative ventilation or intra-aortic balloon pump (IABP). The time between catheterisation and surgery was grouped as: within 24 hours; $>24$ hours for this admission; or $>24$ hours for a previous admission. Information was obtained on triple vessel disease, left main stem disease, ejection fraction (grouped as: good $\geq 50 \%$; fair 30 to $49 \%$; or poor $<30 \%$ ), operative priority (elective, urgent and emergency/salvage). Finally the cardiac procedures being undertaken were classified as coronary artery bypass graft surgery (CAGB) only; valve only; or CABG and valve and other/multiple. Procedures classed as other included major aortic surgery; left ventricular aneurysmectomy; atrial myxoma surgery; pulmonary embolectomy; epicardial pacemaker placement; pericardectomy; atrial septal defect closure; procedure for congenital conditions; acquired ventricular septal defect closure; pulmonary endarterectomy; atrial fibrillation ablation; myomectomy; cardiac surgery plus carotid endarterectomy or peripheral vascular procedures; or any other cardiothoracic procedure not listed above.

\section{Outcomes following surgery}

Information on pulmonary complications (reintubation and ventilation; full tracheostomy; need for CPAP or BIPAP; or prolonged ventilation $>48$ hours), infectious complications (sternal wound infection; leg wound infection; chest infection; or septicaemia or other infection), length of stay in hospital (in days) and death in hospital was obtained from PATS.

\section{Statistical analyses}

The association between AKI and outcomes following surgery were modelled using logistic regression models. The data from Bristol and Birmingham were used to develop the prognostic models (development sample) and the models were validated on the data from Wolverhampton (validation sample). Any-stage AKI and stage-3 AKI were modelled using separate logistic regression models. Univariable associations were examined for all demographic and preoperative factors. Mutivariable associations were examined by entering all demographic and preoperative factors into a single model, which controlled for all these main effects, in a full model. Factors selected for the initial prognostic model were those from the multivariable full model with $P<0.001$. Factors selected for the more inclusive prognostic model were those from the full model with $P<0.05$. The area under the receiver operating characteristic (ROC) curve (AUC) was calculated for each model in the development and validation samples to quantify diagnostic utility. Model calibration was assessed using the Hosmer-Lemeshow test and calibration plots. Calibration plots of observed versus predicted values for AKI were analysed using linear regression to provide the slope and intercept where the closer the intercept to 0 , and the closer the slope to 1 , the better the calibration. The discrimination and calibration of the models were compared to two published AKI risk scores from US data [7,8], a recently published risk score from Australia [10] as well as a mortality risk score - the logistic Euroscore [18]. We were able to match all the variables for the Cleveland clinic score [7] except congestive heart failure and left ventricular ejection fraction $<35 \%$ (data cut at $<30 \%$ ). For the $\mathrm{Ng}$ score [10] we were able to match all the variables with the exception of infective endocarditis. For the Mehta score [8] we were unable to match ethnicity in the development sample, and were unable to precisely match the field MI within 3 weeks, or the type of valve procedure (mitral versus aortic).

We conducted sensitivity analyses, first by considering the inclusion of two-way interactions in our score, and second, by using multiple imputations by chained equations $[19,20]$ to account for missing data. The analysis assumes any systematic difference between the missing values and the observed values can be explained by differences in observed data. We used the ice command [21] in Stata to impute confounder and outcome missing data. Variables that may help explain the missing data (for example, demographic information, preoperative factors, AKI status and outcomes following surgery) were included in the imputation model. The missing 
Table 1 Number and percentage missing for each variable by centre

\begin{tabular}{|c|c|c|c|c|c|c|}
\hline \multirow[b]{2}{*}{ Variable } & \multicolumn{2}{|c|}{ Bristol $(n=18,686)$} & \multicolumn{2}{|c|}{ Birmingham $(n=7,306)$} & \multicolumn{2}{|c|}{ Wovlerhampton $(n=4,862)$} \\
\hline & Number & $\%$ & Number & $\%$ & Number & $\%$ \\
\hline Age & 0 & 0.0 & 0 & 0.0 & 0 & 0.0 \\
\hline Sex & 2 & 0.0 & 0 & 0.0 & 0 & 0.0 \\
\hline Body mass index & 341 & 1.8 & 29 & 0.4 & 1 & 0.0 \\
\hline Smoking status & 114 & 0.6 & 28 & 0.4 & 1 & 0.0 \\
\hline Angina & 79 & 0.4 & 15 & 0.2 & 0 & 0.0 \\
\hline Dyspnoea & 93 & 0.5 & 20 & 0.3 & 0 & 0.0 \\
\hline Previous Ml & 54 & 0.3 & 37 & 0.5 & 10 & 0.2 \\
\hline Previous operations & 15 & 0.1 & 9 & 0.1 & 0 & 0.0 \\
\hline Preoperative diabetes & 75 & 0.4 & 15 & 0.2 & 0 & 0.0 \\
\hline Peripheral vascular disease & 67 & 0.4 & 16 & 0.2 & 0 & 0.0 \\
\hline Pulmonary disease & 65 & 0.4 & 18 & 0.3 & 0 & 0.0 \\
\hline Neurological disease & 59 & 0.3 & 14 & 0.2 & 0 & 0.0 \\
\hline Hypertension & 130 & 0.7 & 29 & 0.4 & 1 & 0.0 \\
\hline Haemoglobin & 1,298 & 7.0 & 2,846 & 39.0 & 28 & 0.6 \\
\hline Baseline serum creatinine & 282 & 1.5 & 66 & 0.9 & 19 & 0.4 \\
\hline GFR & 537 & 2.9 & 86 & 1.2 & 19 & 0.4 \\
\hline Heparin or nitrates & 51 & 0.3 & 18 & 0.3 & 0 & 0.0 \\
\hline Critical preoperative event & 0 & 0.0 & 12 & 0.2 & 0 & 0.0 \\
\hline Catheterisation to surgery & 1,447 & 7.7 & 398 & 5.5 & 242 & 5.0 \\
\hline Triple vessel disease & 938 & 5.0 & 252 & 3.5 & 85 & 1.8 \\
\hline Left main stem disease & 2,286 & 12.2 & 245 & 3.4 & 83 & 1.7 \\
\hline Ejection fraction & 493 & 2.6 & 86 & 1.2 & 0 & 0.0 \\
\hline Operative priority & 23 & 0.1 & 0 & 0.0 & 0 & 0.0 \\
\hline Cardiac procedures & 0 & 0.0 & 0 & 0.0 & 0 & 0.0 \\
\hline Postoperative creatinine & 282 & 1.5 & 66 & 0.9 & 84 & 1.7 \\
\hline Acute kidney injury & 629 & 3.4 & 215 & 2.9 & 84 & 1.7 \\
\hline Acute kidney injury stage & 629 & 3.4 & 215 & 2.9 & 84 & 1.7 \\
\hline Postoperative stay & 10 & 0.1 & 0 & 0.0 & 0 & 0.0 \\
\hline Infective complication & 1,564 & 8.4 & 61 & 0.8 & 96 & 2.0 \\
\hline Pulmonary complication & 1,651 & 8.8 & 53 & 0.7 & 100 & 2.1 \\
\hline Died in hospital & 1 & 0.0 & 6 & 0.1 & 0 & 0.0 \\
\hline Euroscore & 129 & 0.7 & 48 & 0.7 & 6 & 0.1 \\
\hline Cleveland clinic score* & 731 & 3.9 & 139 & 1.9 & 19 & 0.4 \\
\hline Mehta score* & 369 & 2.0 & 101 & 1.4 & 29 & 0.6 \\
\hline Ng score* & 613 & 3.3 & 92 & 1.3 & 20 & 0.4 \\
\hline
\end{tabular}

*Approximation to scores, matching where data are available. MI, myocardial infaction.

values were sampled from their predictive distribution, based on the observed data. Standard regression analyses were used to fit the model of interest to each of the imputed datasets. Ten cycles of regression were carried out and 20 datasets imputed. All 20 estimated associations were combined to give one overall estimated association of interest. Standard error was calculated using the Rubin rules [22,23]. These rules took into account the variability in results between the imputed datasets, indicating the uncertainty of the missing values. All analyses were carried out in StataTM version 13.

\section{Online calculator}

We constructed a web-based calculator for the any-stage AKI risk score [24]. 
Table 2 Characteristics of patients by centre in the complete case data

\begin{tabular}{|c|c|c|c|}
\hline & $\begin{array}{l}\text { Bristol }(n=12,435) \\
\text { Number }(\%)\end{array}$ & $\begin{array}{l}\text { Birmingham }(n=4,092) \\
\text { Number }(\%)\end{array}$ & $\begin{array}{l}\text { Wolverhampton }(n=4,468) \\
\text { Number }(\%)\end{array}$ \\
\hline \multicolumn{4}{|c|}{ Demographic factors } \\
\hline \multicolumn{4}{|l|}{ Age, years } \\
\hline$<60$ & 2,995 (24.1) & $1,061(25.9)$ & $1,026(23.0)$ \\
\hline 60 to 74 & $6,873(55.3)$ & $2,201(53.8)$ & $2,471(55.3)$ \\
\hline$\geq 75$ & 2,567 (20.6) & $830(20.3)$ & $971(21.7)$ \\
\hline \multicolumn{4}{|l|}{ Sex } \\
\hline Male & $9,474(76.2)$ & $2,990(73.1)$ & $3,420(76.5)$ \\
\hline Female & 2,961 (23.8) & $1,102(26.9)$ & $1,048(23.5)$ \\
\hline \multicolumn{4}{|l|}{ Body mass index } \\
\hline$<20.0$ & $482(3.9)$ & $112(2.7)$ & $87(1.9)$ \\
\hline 20.0 to 24.9 & $3,206(25.8)$ & $1,003(24.5)$ & $1,057(23.7)$ \\
\hline 25.0 to 29.9 & $5,685(45.7)$ & $1,737(42.4)$ & $2,020(45.2)$ \\
\hline 30.0 to 34.9 & 2,365 (19.0) & $893(21.8)$ & 967 (21.6) \\
\hline $35.0+$ & $697(5.6)$ & $347(8.5)$ & $337(7.5)$ \\
\hline \multicolumn{4}{|l|}{ Smoking status } \\
\hline Never smoked & 3,970 (31.9) & $1,351(33.0)$ & $1,633(36.5)$ \\
\hline Ex-smoker & $7,072(56.9)$ & $2,427(59.3)$ & $2,414(54.0)$ \\
\hline Current smoker & 1,393 (11.2) & $314(7.7)$ & $421(9.4)$ \\
\hline \multicolumn{4}{|c|}{ Preoperative factors } \\
\hline \multicolumn{4}{|l|}{ Angina $^{a}$} \\
\hline No angina & 2,346 (18.9) & $737(18.0)$ & $779(17.4)$ \\
\hline $\operatorname{CCS} 1$ & $1,460(11.7)$ & $399(9.8)$ & $244(5.5)$ \\
\hline $\operatorname{cCS} 2$ & $3,611(29.0)$ & $1,354(33.1)$ & $1,342(30.0)$ \\
\hline $\operatorname{cCS} 3$ & 2,885 (23.2) & $949(23.2)$ & $1,142(25.6)$ \\
\hline $\operatorname{cCS} 4$ & 2,133 (17.2) & $653(16.0)$ & $961(21.5)$ \\
\hline \multicolumn{4}{|l|}{ Dyspnoea $^{b}$} \\
\hline NYHA 1 & $2,797(22.5)$ & $786(19.2)$ & $926(20.7)$ \\
\hline NHYA 2 & $5,129(41.2)$ & $2,094(51.2)$ & $1,748(39.1)$ \\
\hline NYHA 3 & 3,895 (31.3) & $928(22.7)$ & 1,353 (30.3) \\
\hline NYHA 4 & $614(4.9)$ & $284(6.9)$ & $441(9.9)$ \\
\hline \multicolumn{4}{|l|}{ Previous Ml } \\
\hline 0 & $7,786(62.6)$ & $2,671(65.3)$ & $2,453(54.9)$ \\
\hline 1 & $3,783(30.4)$ & $1,145(28.0)$ & $1,620(36.3)$ \\
\hline$\geq 2$ & $866(7.0)$ & $276(6.7)$ & $395(8.8)$ \\
\hline \multicolumn{4}{|l|}{ Previous operations } \\
\hline 0 & $1,1940(96.0)$ & 3,895 (95.2) & 4,352 (97.4) \\
\hline $1+$ & $495(4.0)$ & $197(4.8)$ & $116(2.6)$ \\
\hline \multicolumn{4}{|c|}{ Preoperative diabetes } \\
\hline No & $10,243(82.4)$ & $3,176(77.6)$ & 3,399 (76.1) \\
\hline Yes & 2,192 (17.6) & $916(22.4)$ & $1,069(23.9)$ \\
\hline \multicolumn{4}{|c|}{ Peripheral vascular disease } \\
\hline No & $11,388(91.6)$ & $3,536(86.4)$ & $3,665(82.0)$ \\
\hline Yes & $1,047(8.4)$ & $556(13.6)$ & $803(18.0)$ \\
\hline
\end{tabular}


Table 2 Characteristics of patients by centre in the complete case data (Continued)

\section{Pulmonary disease}

No

Yes

Neurological disease

$\begin{array}{ll}\text { No } & 11,303(90.9) \\ \text { Yes } & 1,132(9.1)\end{array}$

Hypertension $^{c}$

No

Yes

Haemoglobin

$\begin{array}{ll}<10.0 & 218(1.8) \\ 10.0 \text { to } 11.9 & 1,487(12.0) \\ 12.0+ & 10,730(86.3) \\ \text { GFR } & \\ <30.0 & 436(3.5) \\ 30.0 \text { to } 59.9 & 4,592(36.9) \\ 60.0 \text { to } 89.9 & 5,458(43.9) \\ 90.0+ & 1,949(15.7)\end{array}$

Heparin or nitrates

$\begin{array}{ll}\text { None } & 10,724(86.2) \\ \text { Within a week } & 914(7.4) \\ \text { At operation } & 797(6.4)\end{array}$

Critical preoperative event

$\begin{array}{ll}\text { No } & 12,268(98.7) \\ \text { Yes } & 167(1.3)\end{array}$

Catheter to surgery

Within $24 \mathrm{~h}$

$>24 \mathrm{~h}$ this admission

$>24 \mathrm{~h}$ previous admission

Triple vessel disease

No
Yes

Left main stem disease

No
Yes
Ejection fraction
Good (50+ \%)
Fair (30 to 49\%)
Poor (<30\%)

Operative priority

Elective
Urgent
Emergency/salvage

$$
4,400(35.4)
$$$$
8,035 \text { (64.6) }
$$

$$
\begin{aligned}
& 218(1.8) \\
& 1,487(12.0)
\end{aligned}
$$

(167.3)

$$
299 \text { (2.4) }
$$$$
\text { 6,825 (54.9) }
$$$$
5,311(42.7)
$$

6,041 (48.6)

$$
\text { 6,394 (51.4) }
$$

$10,208(82.1)$

2,227 (17.9)

9,111 (73.3)

2,668 (21.5)

656 (5.3)

$7,350(59.1)$

4,896 (39.4)

189 (1.5)
3,499 (85.5)

593 (14.5)

$3,926(87.9)$

542 (12.1)

3,790 (92.6)

4,077 (91.2)

$302(7.4)$

$391(8.8)$

1,307 (31.9)

1,673 (37.4)

2,785 (68.1)

2,795 (62.6)

91 (2.2)

71 (1.6)

593 (14.5)

515 (11.5)

3,408 (83.3)

3,882 (86.9)

$102(2.5)$

54 (1.2)

$1,460(35.7)$

1,059 (23.7)

1,741 (42.5)

$1,887(42.2)$

789 (19.3)

1,468 (32.9)

3,595 (87.9)

4,133 (92.5)

$53(1.3)$

114 (2.6)

444 (10.9)

221 (4.9)

3,944 (96.4)

4,392 (98.3)

$148(3.6)$

$76(1.7)$

$38(0.9)$

84 (1.9)

1,019 (24.9)

971 (21.7)

$3,035(74.2)$

$3,413(76.4)$

1,759 (43.0)

$1,819(40.7)$

2,333 (57.0)

2,649 (59.3)

3,162 (77.3)

3,422 (76.6)

930 (22.7)

1,046 (23.4)

2,758 (67.4)

2,910 (65.1)

$1,143(27.9)$

$1,192(26.7)$

191 (4.7)

366 (8.2)

2,798 (68.4)

3,173 (71.0)

$1,256(30.7)$

1,255 (28.1)

$38(0.9)$

40 (0.9) 
Table 2 Characteristics of patients by centre in the complete case data (Continued)

\begin{tabular}{|c|c|c|c|}
\hline \multicolumn{4}{|l|}{ Cardiac procedures } \\
\hline CABG only & $8,587(69.1)$ & $2,241(54.8)$ & 2,974 (66.6) \\
\hline Valve only & $1,838(14.8)$ & $644(15.7)$ & $622(13.9)$ \\
\hline CABG+valve & $1,169(9.4)$ & $554(13.5)$ & $568(12.7)$ \\
\hline Other/multiple & $841(6.8)$ & $653(16.0)$ & $304(6.8)$ \\
\hline \multicolumn{4}{|c|}{ Acute kidney injury, \% } \\
\hline None & $9,495(76.4)$ & 3,238 (79.1) & $3,370(75.4)$ \\
\hline Stage 1 & $2,164(17.4)$ & $560(13.7)$ & $706(15.8)$ \\
\hline Stage 2 & $330(2.7)$ & $89(2.2)$ & $124(2.8)$ \\
\hline Stage 3 & $446(3.6)$ & $205(5.0)$ & $268(6.0)$ \\
\hline
\end{tabular}

${ }^{a}$ Canadian Cardiovascular Society (CCS) classes: 1 ordinary activity does not cause angina; 2 slight limitation; 3 marked limitation; 4 inability to carry out any physical activity without discomfort. ${ }^{b} \mathrm{New}$ York Heart Association (NYHA) functional classification: 1 no symptoms and no limitation in ordinary physical activity; 2 mild symptoms and slight limitation during ordinary activity; 3 marked limitation in activity due to symptoms, comfortable only at rest; 4 severe limitations, experiences symptoms even while at rest. ' Hypertension was defined as treated or blood pressure $>140 / 90$. CABG, coronary artery bypass graft; GFR, glomerular filtration rate; Haemoglobin, $\mathrm{g} / \mathrm{dL} ; \mathrm{GFR}, \mathrm{mL} / \mathrm{min}$.

\section{Results}

\section{Characteristics of patients}

Data were available from 18,686 patients from Bristol and 7,306 patients from Birmingham (the developmental sample) and 4,862 patients from Wolverhampton (the validation sample). Missing data for each centre are reported in Table 1. The most common field that was missing was the baseline $\mathrm{Hb}$. This was missing in $39 \%$ of the Birmingham data, and predominantly in the years prior to 2004. Emergency/salvage surgery were more likely to have missing preoperative creatinine (Bristol 10\%, Birmingham 14\%, Wolverhampton 3\% missing) compared to elective or urgent surgery (Bristol 1\%, Birmingham 1\%, Wolverhampton $0 \%$ missing). The complete case analysis included 20,995 patients, of whom 12,435 were from Bristol, 4,092 were from Birmingham and 4,468 were from Wolverhampton (Table 2).

In the complete case data median age by centre was: Bristol 68 years (IQR 60,74), Birmingham 67 years $(59,73)$ and Wolverhampton 67 years $(60,74)$. The majority of operations were performed on men (76.2\% in Bristol, 73.1\% in Birmingham and $76.5 \%$ in Wolverhampton). The most common procedure was CABG only $(69.1 \%$ in Bristol, $54.8 \%$ in Birmingham and $66.6 \%$ in Wolverhampton). Mean (SD) baseline creatinine values by centre were Bristol 108.9 $\mu \mathrm{mol} / \mathrm{L}$ (28.5), Birmingham 107.1 $\mu \mathrm{mol} / \mathrm{L}$ (34.4) and Wolverhampton $94.8 \mu \mathrm{mol} / \mathrm{L}$ (28.4) Any stage of AKI affected $23.7 \%$ patients in the Bristol dataset, $20.9 \%$ patients in Birmingham and $24.6 \%$ patients in Wolverhampton. Stage-3 AKI was slightly more prevalent in the validation dataset $(6.0 \%)$ compared to the development sample (3.6\% in Bristol and 5.0\% in Birmingham). The criteria by which patients were defined as having AKI are described in Table 3.

\section{KDIGO-defined AKI severity and outcome}

AKI was associated with increased hospital stay (Table 4). Patients without AKI had a median postoperative hospital stay of 6 days in Bristol (IQR 5, 8) and Wolverhampton $(5,7)$, and 9 days $(6,13)$ in Birmingham (Table 4).

Table 3 Total patients who met different acute kidney injury (AKI) criteria in the complete case data

\begin{tabular}{|c|c|c|c|c|c|}
\hline & \multirow{3}{*}{$\begin{array}{l}\text { Ratio of postoperative to } \\
\text { preoperative creatinine }\end{array}$} & \multirow{2}{*}{\multicolumn{2}{|c|}{$\begin{array}{l}\text { Postoperative creatinine }<354 \\
\text { Difference from baseline }\end{array}$}} & \multicolumn{2}{|c|}{ Postoperative creatinine $\geq 354$} \\
\hline & & & & & \\
\hline & & $<26$ & $\geq 26$ & $<26$ & $\geq 26$ \\
\hline \multirow[t]{4}{*}{ No RRT } & $<1.5$ & $16,094^{a}$ & $2,152^{b}$ & $9^{a}$ & $11^{d}$ \\
\hline & 1.5 to 1.9 & $1^{\mathrm{b}}$ & $1,277^{b}$ & & $33^{d}$ \\
\hline & 2.0 to 2.9 & & $543^{c}$ & & $94^{d}$ \\
\hline & $\geq 3.0$ & & $81^{d}$ & & $95^{d}$ \\
\hline \multirow[t]{4}{*}{ Commenced on RRT } & $<1.5$ & $77^{d}$ & $71^{d}$ & & $4^{d}$ \\
\hline & 1.5 to 1.9 & & $137^{d}$ & & $7^{d}$ \\
\hline & 2.0 to 2.9 & & $155^{d}$ & & $34^{d}$ \\
\hline & $\geq 3.0$ & & $43^{d}$ & & $77^{d}$ \\
\hline
\end{tabular}

Creatinine measure in $\mu \mathrm{mol} / \mathrm{L} .{ }^{\mathrm{a}} \mathrm{No} \mathrm{AKI}$, total $=16,103 ;{ }^{\mathrm{b}}$ stage-1 AKI, total $=3,430 ;{ }^{\mathrm{c}}$ stage-2 AKI, total $=543 ;{ }^{d}$ stage-3 AKI, total $=919 . \mathrm{RRT}$, renal replacement therapy. 
Table 4 Length of stay following surgery by acute kidney injury (AKI) status

\begin{tabular}{|c|c|c|c|c|c|c|c|c|c|}
\hline & \multicolumn{3}{|l|}{ Bristol } & \multicolumn{3}{|c|}{ Birmingham } & \multicolumn{3}{|c|}{ Wolverhampton } \\
\hline & Numbe & Median & IQR & Number & Median & IQR & Number & Median & IQR \\
\hline \multicolumn{10}{|c|}{ All patients } \\
\hline \multicolumn{10}{|l|}{ AKI } \\
\hline None & 9495 & 6 & $(5,8)$ & 3238 & 9 & $(6,13)$ & 3370 & 6 & $(5,7)$ \\
\hline Stage 1 & 2164 & 9 & $(7,13)$ & 560 & 13 & $(9,19)$ & 706 & 8 & $(6,13)$ \\
\hline Stage 2 & 330 & 11 & $(8,16)$ & 89 & 19 & $(12,32)$ & 124 & 10 & $(8,17)$ \\
\hline Stage 3 & 446 & 14 & $(10,26)$ & 205 & 19 & $(10,42)$ & 268 & 16 & $(9,32)$ \\
\hline \multicolumn{10}{|c|}{ Patients who did not die in hospital } \\
\hline \multicolumn{10}{|l|}{ AKI } \\
\hline None & 9464 & 6 & $(5,8)$ & 3205 & 9 & $(7,13)$ & 3357 & 6 & $(5,7)$ \\
\hline Stage 1 & 2119 & 9 & $(7,13)$ & 544 & 13 & $(9,18)$ & 693 & 8 & $(6,13)$ \\
\hline Stage 2 & 313 & 11 & $(8,16)$ & 88 & 19 & $(12,32)$ & 122 & 10 & $(8,17)$ \\
\hline Stage 3 & 338 & 15 & $(10,27)$ & 133 & 25 & $(14,43)$ & 190 & 17 & $(11,32)$ \\
\hline
\end{tabular}

For patients with stage-1 AKI, median postoperative stay increased to 9 days (IQR 7,13$)$ in Bristol, $13(9,19)$ in Birmingham and $8(6,13)$ in Wolverhampton. Patients with the most severe AKI (stages 2 and 3) had the longest postoperative stay. In the complete case data $(n=20,995)$ stage-1 AKI was associated with higher odds of infective and pulmonary complications and an almost five-fold increase in the odds of death in hospital (Table 5). Odds ratios for stage-3 AKI compared to no AKI were 7.6 (95\% CI $6.6,8.8)$ for infective complications, $9.4(8.2,10.8)$ for pulmonary complications and 81.2 $(62.3,106.0)$ for death in hospital. Over $80 \%$ of all deaths in hospital were preceded by AKI.

\section{Model building for the clinical prediction score}

A total of 23 factors were examined for their associations with AKI. Odds ratios for unadjusted and fully adjusted models are presented in Table 6 (for models of any-stage AKI) and Table 7 (for models of stage-3 AKI). In fully adjusted models, 15 factors were strongly associated with any-stage AKI $(P<0.001)$ and were included in the initial prognostic model. A further five factors were associated with any-stage AKI at the level of $P<0.05$, and were added to the more inclusive model. For stage-3 AKI, eight factors were included in the initial prognostic model $(P<0.001)$, a further three factors were included in the more inclusive prognostic model $(P<0.05)$.

\section{Diagnostic utility}

\section{Any-stage AKI score}

The ROC AUC for the initial model for any-stage AKI in the development sample was 0.73 (95\% CI 0.72, 0.74; Figure 1 and Table 8 ) and was very similar in the validation sample (AUC 0.74; 95\% CI 0.72, 0.76). The Hosmer Lemshow test $(P=0.490$, Table 9$)$ and plot of observed versus predicted any-stage AKI (Figure 2A and Table 9) demonstrated good calibration in the development sample. The initial model was less well-calibrated in the validation sample (Hosmer Lemshow $P=0.192$, and Figure 2B). The more inclusive model did not demonstrate better discrimination when compared to the initial model (Table 8), however, it did demonstrate better calibration as evidenced by the Hosmer-Lemeshow test $(P=0.406$ in the validation sample) and plots of observed versus predicted any-stage AKI (Figure 2B and Table 9).

For the validation sample discrimination by the any-stage AKI score was better than the Euroscore (AUC 0.68; 95\% CI 0.67, 0.70) and Cleveland clinic (AUC 0.70; 95\% CI 0.69,

Table 5 Outcomes following surgery by acute kidney injury (AKI) status, data from all centres

\begin{tabular}{|c|c|c|c|c|c|c|c|}
\hline \multirow[t]{2}{*}{ AKI status } & \multirow[t]{2}{*}{ Total number } & \multicolumn{2}{|c|}{ Infective complication } & \multicolumn{2}{|c|}{ Pulmonary complication } & \multicolumn{2}{|c|}{ Died in hospital } \\
\hline & & Number (\%) & Odds ratio $(95 \% \mathrm{CI})$ & Number (\%) & Odds ratio $(95 \% \mathrm{Cl})$ & Number (\%) & Odds ratio $(95 \% \mathrm{Cl})$ \\
\hline None & 16103 & $1430(8.9)$ & 1 & $1810(11.2)$ & 1 & $77(0.5)$ & 1 \\
\hline Stage 1 & 3430 & $623(18.2)$ & $2.3(2.1,2.5)$ & $793(23.1)$ & $2.4(2.2,2.6)$ & $74(2.2)$ & $4.6(3.3,6.3)$ \\
\hline Stage 2 & 543 & $133(24.5)$ & $3.3(2.7,4.1)$ & $192(35.4)$ & $4.3(3.6,5.2)$ & $20(3.7)$ & $8.0(4.8,13.1)$ \\
\hline Stage 3 & 919 & $392(42.7)$ & $7.6(6.6,8.8)$ & $500(54.4)$ & $9.4(8.2,10.8)$ & $258(28.1)$ & $81.2(62.3,106.0)$ \\
\hline$P$-value & & & $<0.001$ & & $<0.001$ & & $<0.001$ \\
\hline
\end{tabular}

$P$-values for trend derived from logistic regression models. 
Table 6 Associations of preoperative factors with any-stage acute kidney injury (AKI) in the development sample (Bristol and Birmingham)

\begin{tabular}{|c|c|c|c|c|c|c|c|c|}
\hline & \multirow{2}{*}{$\begin{array}{l}\text { Number } \\
\text { Total }\end{array}$} & \multirow{2}{*}{$\begin{array}{l}\text { Number (\%) } \\
\text { Any AKI }\end{array}$} & \multicolumn{3}{|l|}{ Unadjusted } & \multicolumn{3}{|c|}{ Fully adjusted } \\
\hline & & & Odds ratio & $95 \% \mathrm{Cl}$ & $P$-value & Odds ratio & $95 \% \mathrm{Cl}$ & $P$-value \\
\hline \multicolumn{9}{|c|}{ Demographic factors } \\
\hline \multicolumn{9}{|l|}{ Age, years } \\
\hline$<60$ & 4,056 & $485(12.0)$ & 1.00 & & & 1.00 & & \\
\hline 60 to 74 & 9,074 & $2,039(22.5)$ & 2.13 & $(1.92,2.37)$ & & 1.53 & $(1.35,1.74)$ & \\
\hline$\geq 75$ & 3,397 & $1,270(37.4)$ & 4.40 & $(3.91,4.94)$ & $<0.001$ & 2.21 & $(1.91,2.57)$ & $<0.001$ \\
\hline \multicolumn{9}{|l|}{ Sex } \\
\hline Male & 12,464 & 2,891 (23.2) & 1.00 & & & 1.00 & & \\
\hline Female & 4,063 & $903(22.2)$ & 0.95 & $(0.87,1.03)$ & 0.202 & 0.53 & $(0.48,0.59)$ & $<0.001$ \\
\hline \multicolumn{9}{|l|}{ Body mass index } \\
\hline$<20.0$ & 594 & $175(29.5)$ & 1.60 & $(1.33,1.92)$ & & 0.80 & $(0.64,0.99)$ & \\
\hline 20.0 to 24.9 & 4,209 & $931(22.1)$ & 1.08 & $(0.99,1.19)$ & & 0.79 & $(0.71,0.88)$ & \\
\hline 25.0 to -29.9 & 7,422 & $1,540(20.7)$ & 1.00 & & & 1.00 & & \\
\hline 30.0 to 34.9 & 3,258 & $854(26.2)$ & 1.36 & $(1.23,1.49)$ & & 1.75 & $(1.57,1.95)$ & \\
\hline $35.0+$ & 1,044 & $294(28.2)$ & 1.50 & $(1.29,1.73)$ & $<0.001$ & 2.11 & $(1.78,2.50)$ & $<0.001$ \\
\hline \multicolumn{9}{|l|}{ Smoking status } \\
\hline Never smoked & 5,321 & $1,122(21.1)$ & 1.00 & & & 1.00 & & \\
\hline Ex-smoker & 9,499 & 2,315 (24.4) & 1.21 & $(1.11,1.31)$ & & 1.09 & $(1.00,1.20)$ & \\
\hline Current smoker & 1,707 & 357 (20.9) & 0.99 & $(0.87,1.13)$ & $<0.001$ & 1.41 & $(1.22,1.64)$ & $<0.001$ \\
\hline
\end{tabular}

\section{Preoperative factors}

Angina

No angina
CCS 1
CCS 2
CCS 3
CCS 4

Dyspnoea

NYHA 1

NHYA 2

NYHA 3

NYHA 4

Previous Ml

0

1

$\geq 2$

Previous operations

0

$1+$

Preoperative diabetes

No
Yes

Yes

$\begin{array}{lll}3,083 & 810(26.3) & 1.00 \\ 1,859 & 409(22.0) & 0.79 \\ 4,965 & 994(20.0) & 0.70 \\ 3,834 & 870(22.7) & 0.82 \\ 2,786 & 711(25.5) & 0.96\end{array}$

3,583

7,223

4,823

898

10,457

4,928

$2,272(21.7)$

$1,174(23.8)$

1.13

1,142

$348(30.5)$

1.58

15,835

3,578 (22.6)

1.00

692

$216(31.2)$

1.55

13,419

2,824 (21.0)

1.00

3,108

$970(31.2)$

1.70
$(0.69,0.91)$

$(0.63,0.78)$

$(0.74,0.92)$

$(0.86,1.08)$

$<0.001$

$\begin{array}{lll} & & 1.00 \\ (1.12,1.38) & & 1.07 \\ (1.72,2.13) & & 1.29 \\ (2.77,3.81) & <0.001 & 1.37\end{array}$

$(0.95,1.19)$

$(1.14,1.46)$

$(1.13,1.66)$

$<0.001$

$(1.04,1.22)$

$(1.38,1.81)$

$<0.001$

1.00

0.99

$(0.90,1.10)$

$(0.88,1.21)$

0.879

$\begin{array}{lll}(1.32,1.83) & <0.001 \quad 1.39\end{array}$

$(1.15,1.67)$

0.007

1.00

$(1.56,1.86) \quad<0.001 \quad 1.41$ 
Table 6 Associations of preoperative factors with any-stage acute kidney injury (AKI) in the development sample (Bristol and Birmingham) (Continued)

Peripheral vascular disease

$\begin{array}{llll}\text { No } & 14,924 & 3,237(21.7) & 1.00 \\ \text { Yes } & 1,603 & 557(34.7) & 1.92\end{array}$

Pulmonary disease

$$
\text { No }
$$$$
\text { Yes }
$$

Neurological disease

$$
\begin{aligned}
& \text { No } \\
& \text { Yes }
\end{aligned}
$$

Hypertension

$$
\begin{aligned}
& \text { No } \\
& \text { Yes }
\end{aligned}
$$

Haemoglobin

$$
\begin{aligned}
& <10.0 \\
& 10.0 \text { to11.9 }
\end{aligned}
$$$$
12.0+
$$

Glomerular filtration rate

$<30.0$
30.0 to 59.9
60.0 to 89.9

Heparin or nitrates

Wone

At operation

Critical preoperative event

$$
\begin{aligned}
& \text { No } \\
& \text { Yes }
\end{aligned}
$$

Catheter to surgery

$$
\begin{aligned}
& \text { Within } 24 \mathrm{~h} \\
& >24 \mathrm{~h} \text { this admission }
\end{aligned}
$$

$>24 \mathrm{~h}$ prev admission

Triple vessel disease

$$
\begin{aligned}
& \text { No } \\
& \text { Yes }
\end{aligned}
$$

Left main stem disease

$$
\begin{aligned}
& \text { No } \\
& \text { Yes }
\end{aligned}
$$

Ejection fraction

$$
\begin{aligned}
& \text { Good (50+ \%) } \\
& \text { Fair (30 to 49\%) } \\
& \text { Poor (<30\%) }
\end{aligned}
$$

$\begin{array}{lll}14,490 & 3,217(22.2) & 1.00 \\ 2,037 & 577(28.3) & 1.38\end{array}$

15,093

$3,346(22.2)$

1.00

1,434

$448(31.2)$

1.60

5,707

10,820

$1,028(18.0)$

$2,766(25.6)$

1.00

1.56

309

2,080

133 (43.0)

2.98

800 (38.5)

2.46

14,138

$2,861(20.2)$

1.00

$538 \quad 283(52.6) \quad 5.64$

6,052

$1,917(31.7)$

2.36

7,199

$1,184(16.4)$

1.00

2,738

$410(15.0)$

0.89

14,319

$3,086(21.6)$

1.00

$$
967
$$

299 (30.9)

1.63

1,241

409 (33.0)

1.79

16,212

$3,640(22.5)$

315

154 (48.9)

1.00

337

7,844

125 (37.1)

1.95

$1,730(22.1)$

0.93

8,346

$1,939(23.2)$

1.00

7,800

1,697 (21.8)

2,097 (24.0)

1.00

8,727

1.14

13,370

3,014 (22.5)

3,157

780 (24.7)

1.00

1.13

11,869

2,362 (19.9)

1.00

3,811

1,121 (29.4)

1.68

847

311 (36.7)

2.34 $\begin{array}{lll} & 1.00 \\ (1.72,2.15) \quad<0.001 & 1.31\end{array}$

$(1.16,1.48)$

$<0.001$

$(1.25,1.54) \quad<0.001 \quad 1.07$

$(0.96,1.20)$

0.240

$(1.42,1.80) \quad<0.001 \quad 1.14$

$(1.00,1.30)$

0.044

$\begin{array}{ll} & 1.00 \\ (1.44,1.69) & <0.001\end{array}$

$(2.37,3.74)$

$(2.23,2.72)$

1.58
1.67
$<0.001 \quad 1.00$

$(1.17,1.40)$

$<0.001$

$(1.23,2.04)$

$(1.49,1.87)$

$<0.001$

$(4.71,6.75)$

$(2.17,2.56) \quad 2.25$

$(3.73,5.77)$

$(2.03,2.48)$

$\begin{array}{lll}(0.79,1.01) & <0.001 \quad 0.82\end{array}$

$(0.71,0.95)$

$<0.001$

1.00

$(1.41,1.88) \quad 1.31$

$(1.58,2.03) \quad<0.001 \quad 1.27$

$(1.11,1.55)$

$(1.08,1.48)$

0.001

$(2.64,4.13) \quad<0.001 \quad 1.57$

$(1.19,2.06)$

0.002

$(1.55,2.44)$

$(0.87,1.01)$

1.17

$(0.87,1.57)$

$(0.72,0.88)$

$<0.001$

$<0.001 \quad 1.00$

(

1.00

$(1.06,1.22) \quad<0.001 \quad 1.24$

$(1.12,1.37)$

$<0.001$

$311(36.7)-2.34$

$\begin{array}{lll}(1.54,1.82) & & 1.22 \\ (2.02,2.70) & <0.001 & 1.34\end{array}$

$(1.11,1.34)$

$(1.13,1.59)$

$<0.001$ 
Table 6 Associations of preoperative factors with any-stage acute kidney injury (AKI) in the development sample (Bristol and Birmingham) (Continued)

\begin{tabular}{|c|c|c|c|c|c|c|c|c|}
\hline \multicolumn{9}{|l|}{ Operative priority } \\
\hline Elective & 10,148 & $2,043(20.1)$ & 1.00 & & & 1.00 & & \\
\hline Urgent & 6,152 & $1,644(26.7)$ & 1.45 & $(1.34,1.56)$ & & 1.33 & $(1.20,1.48)$ & \\
\hline Emergency/Salvage & 227 & $107(47.1)$ & 3.54 & $(2.71,4.61)$ & $<0.001$ & 1.93 & $(1.34,2.76)$ & $<0.001$ \\
\hline \multicolumn{9}{|l|}{ Cardiac procedures } \\
\hline CABG only & 10,828 & $2,167(20.0)$ & 1.00 & & & 1.00 & & \\
\hline Valve only & 2,482 & $553(22.3)$ & 1.15 & $(1.03,1.27)$ & & 1.27 & $(1.08,1.48)$ & \\
\hline CABG+valve & 1,723 & $644(37.4)$ & 2.39 & $(2.14,2.66)$ & & 1.81 & $(1.59,2.07)$ & \\
\hline Other/multiple & 1,494 & $430(28.8)$ & 1.62 & $(1.43,1.82)$ & $<0.001$ & 1.62 & $(1.39,1.89)$ & $<0.001$ \\
\hline
\end{tabular}

CCS, Canadian Cardiovascular Society; NYHA, New York Heart Association; Ml, myocardial infarction; prev, previous; CABG, coronary artery bypass graft; Haemoglobin, g/dL; GFR, mL/min.

0.72), and equivalent to the Mehta Score (AUC 0.74; 0.72, 0.76 ) and Ng score (AUC 0.73; 95\% CI 0.71, 0.75) (Table 8). Hosmer-Lemshow tests and calibration plots (Table 9 and Figure $2 \mathrm{~B}$ ) demonstrated that the more inclusive model demonstrated better calibration than each of the four comparison scores.

\section{Stage-3 AKI score}

In the validation sample both the initial prognostic stage-3 AKI model $(0.78,95 \% \mathrm{CI} 0.75,0.80)$ and the more inclusive stage-3 AKI model $(0.79,95 \%$ CI $0.76,0.81)$ demonstrated good discrimination (Figure 1 and Table 8). The stage-3 AKI score had better discrimination than the Euroscore $(0.73 ; 95 \%$ CI $0.70,0.76)$ and similar discrimination to the Cleveland Clinic $(0.78 ; 95 \%$ CI $0.75,0.81)$, Metha score $(0.79 ; 95 \%$ CI $0.77,0.82)$ and Ng Score $(0.79,95 \%$ CI 0.76, 0.82) (Table 8). Plots of observed versus expected stage-3 AKI indicated that the initial stage-3 AKI model had better calibration that each of the four comparison scores, however Hosmer-Lemshow tests suggested that calibration of the stage- 3 score, as well as the four comparison scores was poor $(P<0.001)$ in both development and validation samples (Figure 2C, D).

\section{Final model coefficients}

The model coefficients for the initial model for any-stage AKI and the initial model for stage-3 AKI are shown in Table 10. Factors associated with any-stage AKI in the final model were older age, male sex, BMI $>35 \mathrm{~kg} / \mathrm{m}^{2}$, current smokers, higher dyspnoea categories, diabetes, peripheral vascular disease, hypertension, lower haemoglobin, lower estimated GFR, catheter to surgery within 24 hours, triple vessel disease, poor ejection fraction, emergency/salvage operations and more complex surgery. The only factor included in the stage- 3 model that was not in the any-stage model was a critical preoperative event. Sensitivity analyses showed that adding two-way interactions at $P<0.05$ did not improve the diagnostic utility in the validation sample (data not shown). Further sensitivity analysis using only more recent data (after 2002) did not improve discrimination. Coefficients from imputed data for the final model were very similar to the complete case analysis (data not shown).

\section{Discussion}

This study has developed two new risk scores for the preoperative identification of cardiac surgery patients who are at increased risk of developing AKI. These scores were developed using a large cohort of patients from two UK cardiac centres and externally validated using data from a third UK centre. We compared the diagnostic utility of these scores to four previously published scores; Euroscore, Mehta Score, Cleveland Clinic score and the Ng score. Our risk prediction score for any-stage AKI has demonstrated better discrimination compared to the Euroscore and the Cleveland Clinic Score, and equivalent discrimination to the Mehta and $\mathrm{Ng}$ scores. The any-stage AKI score demonstrated better calibration than the four comparison scores. The stage-3 AKI risk prediction score demonstrated good discrimination, as did the four comparison risk scores, but these scores were less well-calibrated.

The study has important strengths. It has demonstrated the prognostic utility of the KDIGO AKI definition in a large multicentre cohort, and has confirmed the prognostic importance of milder forms of AKI, as has been identified by earlier consensus definitions $[12,13]$. The two risk scores we have developed are the first to our knowledge that have been designed to predict a consensus definition of AKI. The use of consensus AKI definitions as endpoints is a key element of study design that is important for standardisation of reporting and comparative analyses of trials. Furthermore, the any-stage AKI risk score is the first externally validated AKI risk prediction score that has been designed to include patients at risk of KDIGO stage-1 AKI, which we have shown to have prognostic utility in the current study. The any-stage 
Table 7 Associations of preoperative factors with stage-3 acute kidney injury (AKI) in the Bristol and Birmingham development sample (complete case data)

\begin{tabular}{|c|c|c|c|c|c|c|c|c|}
\hline & \multirow{2}{*}{$\begin{array}{l}\text { Number } \\
\text { Total }\end{array}$} & \multirow{2}{*}{$\begin{array}{l}\text { Number (\%) } \\
\text { Stage-3 AKI }\end{array}$} & \multicolumn{3}{|l|}{ Unadjusted } & \multicolumn{3}{|c|}{ Fully adjusted } \\
\hline & & & Odds ratio & $95 \% \mathrm{Cl}$ & $P$-value & Odds ratio & $95 \% \mathrm{Cl}$ & $P$-value \\
\hline \multicolumn{9}{|c|}{ Demographic factors } \\
\hline \multicolumn{9}{|l|}{ Age, years } \\
\hline$<60$ & 4,056 & $75(1.8)$ & 1.00 & & & 1.00 & & \\
\hline 60 to 74 & 9,074 & $352(3.9)$ & 2.14 & $(1.66,2.76)$ & & 1.25 & $(0.94,1.66)$ & \\
\hline$\geq 75$ & 3,397 & $224(6.6)$ & 3.75 & $(2.87,4.89)$ & $<0.001$ & 1.29 & $(0.94,1.78)$ & 0.255 \\
\hline \multicolumn{9}{|l|}{ Sex } \\
\hline Male & 12,464 & $497(4.0)$ & 1.00 & & & 1.00 & & \\
\hline Female & 4,063 & $154(3.8)$ & 0.95 & $(0.79,1.14)$ & 0.575 & 0.45 & $(0.36,0.55)$ & $<0.001$ \\
\hline \multicolumn{9}{|l|}{ Body mass index } \\
\hline$<20.0$ & 594 & $31(5.2)$ & 1.57 & $(1.07,2.31)$ & & 0.54 & $(0.35,0.84)$ & \\
\hline 0.0 to 24.9 & 4,209 & $177(4.2)$ & 1.25 & $(1.03,1.53)$ & & 0.83 & $(0.67,1.03)$ & \\
\hline 25.0 to 29.9 & 7,422 & $251(3.4)$ & 1.00 & & & 1.00 & & \\
\hline 30.0 to 34.9 & 3,258 & $137(4.2)$ & 1.25 & $(1.01,1.55)$ & & 1.79 & $(1.42,2.26)$ & \\
\hline $35.0+$ & 1,044 & $55(5.3)$ & 1.59 & $(1.18,2.14)$ & 0.006 & 2.60 & $(1.85,3.65)$ & $<0.001$ \\
\hline \multicolumn{9}{|l|}{ Smoking status } \\
\hline Never smoked & 5,321 & 193 (3.6) & 1.00 & & & 1.00 & & \\
\hline Ex-smoker & 9,499 & $402(4.2)$ & 1.17 & $(0.99,1.40)$ & & 1.11 & $(0.92,1.35)$ & \\
\hline Current smoker & 1,707 & $56(3.3)$ & 0.90 & $(0.67,1.22)$ & 0.061 & 1.35 & $(0.97,1.88)$ & 0.191 \\
\hline \multicolumn{9}{|c|}{ Pre-operative factors } \\
\hline \multicolumn{9}{|l|}{ Angina } \\
\hline No angina & 3,083 & $176(5.7)$ & 1.00 & & & 1.00 & & \\
\hline CCS 1 & 1,859 & $76(4.1)$ & 0.70 & $(0.53,0.93)$ & & 0.79 & $(0.59,1.06)$ & \\
\hline $\operatorname{cCS} 2$ & 4,965 & $142(2.9)$ & 0.49 & $(0.39,0.61)$ & & 0.68 & $(0.52,0.89)$ & \\
\hline $\operatorname{cCS} 3$ & 3,834 & $135(3.5)$ & 0.60 & $(0.48,0.76)$ & & 0.74 & $(0.56,0.98)$ & \\
\hline $\operatorname{CCS} 4$ & 2,786 & $122(4.4)$ & 0.76 & $(0.60,0.96)$ & $<0.001$ & 0.79 & $(0.59,1.06)$ & 0.047 \\
\hline \multicolumn{9}{|l|}{ Dyspnoea } \\
\hline NYHA 1 & 3,583 & $87(2.4)$ & 1.00 & & & 1.00 & & \\
\hline NHYA 2 & 7,223 & $217(3.0)$ & 1.24 & $(0.97,1.60)$ & & 1.02 & $(0.78,1.32)$ & \\
\hline NYHA 3 & 4,823 & $242(5.0)$ & 2.12 & $(1.66,2.72)$ & & 1.23 & $(0.94,1.63)$ & \\
\hline NYHA 4 & 898 & $105(11.7)$ & 5.32 & $(3.96,7.14)$ & $<0.001$ & 1.47 & $(1.02,2.10)$ & 0.063 \\
\hline \multicolumn{9}{|l|}{ Previous Ml } \\
\hline 0 & 10,457 & 379 (3.6) & 1.00 & & & 1.00 & & \\
\hline 1 & 4,928 & $200(4.1)$ & 1.12 & $(0.94,1.34)$ & & 1.06 & $(0.86,1.31)$ & \\
\hline$\geq 2$ & 1,142 & $72(6.3)$ & 1.79 & $(1.38,2.32)$ & $<0.001$ & 1.18 & $(0.86,1.61)$ & 0.581 \\
\hline \multicolumn{9}{|l|}{ Previous operations } \\
\hline 0 & 15,835 & $596(3.8)$ & 1.00 & & & 1.00 & & \\
\hline $1+$ & 692 & $55(7.9)$ & 2.21 & $(1.66,2.94)$ & $<0.001$ & 1.59 & $(1.15,2.19)$ & 0.006 \\
\hline \multicolumn{9}{|c|}{ Preoperative diabetes } \\
\hline No & 13,419 & $443(3.3)$ & 1.00 & & & 1.00 & & \\
\hline Yes & 3,108 & $208(6.7)$ & 2.10 & $(1.77,2.49)$ & $<0.001$ & 1.80 & $(1.48,2.18)$ & $<0.001$ \\
\hline
\end{tabular}


Table 7 Associations of preoperative factors with stage-3 acute kidney injury (AKI) in the Bristol and Birmingham development sample (complete case data) (Continued)

Peripheral vascular disease

$\begin{array}{llll}\text { No } & 14,924 & 554(3.7) & 1.00 \\ \text { Yes } & 1,603 & 97(6.1) & 1.67\end{array}$

$\begin{array}{ll} & 1.00 \\ (1.34,2.09) \quad<0.001 \quad 1.07\end{array}$

$(0.84,1.37)$

0.582

Pulmonary disease

No
Yes

$\begin{array}{ll}14,490 & 548(3.8) \\ 2,037 & 103(5.1)\end{array}$

$$
1.00
$$

Neurological disease

$$
\begin{aligned}
& \text { No } \\
& \text { Yes }
\end{aligned}
$$$$
15,093
$$$$
579 \text { (3.8) }
$$$$
1.35
$$$$
(1.09,1.68) \quad 0.006
$$$$
1.00
$$

$1,434 \quad 72(5.0)$

$$
\begin{aligned}
& 1.00 \\
& 1.33
\end{aligned}
$$

$(1.03,1.70)$

0.028

1.00

Hypertension

$$
\text { No }
$$

5,707
10,820

$196(3.4)$

$$
1.00
$$$$
455 \text { (4.2) }
$$

$(1.04,1.46)$

0.016

$(4.16,8.07)$

$(3.07,4.36)$

2.34
2.17
$<0.001 \quad 1.00$

$(1.59,3.42)$

$<10.0$

10.0 to 11.9

$309 \quad 45(14.6) \quad 5.79$

2,080

$202(9.7)$

3.66

$12.0+$

14,138

404 (2.9)

1.00

$<0.001$

$(1.78,2.66)$

Glomerular filtration rate

$<30.0$
30.0 to 59.9
60.0 to 89.9

$$
538
$$

98 (18.2)

8.89

6,052

$337(5.6)$

2.35

(6.82, 11.59)

$(1.95,2.83)$

$176(2.4)$

1.00

2,738

40 (1.5)

$$
0.59
$$

$(0.42,0.84)$

1.00

None

14,319

510 (3.6)

$967 \quad 50(5.2)$

1.48

$1,241 \quad 91(7.3)$

$$
2.14
$$

$(1.10,1.99)$

$(1.70,2.70)$

8.64

2.37

1.00

At operation

Critical preoperative event

$$
\begin{aligned}
& \text { No } \\
& \text { Yes }
\end{aligned}
$$$$
16,212
$$$$
591 \text { (3.6) }
$$$$
315
$$

60 (19.0)

1.00

Catheter to surgery

Within $24 \mathrm{~h}$

$>24 \mathrm{~h}$ this admission

337

40 (11.9)

6.22

$(4.64,8.34)$

$$
<0.001
$$$$
2.44
$$

$(1.63,3.66)$

$<0.001$

$>24 \mathrm{~h}$ previous admission

7,844

255 (3.3)

$(2.14,4.28)$

1.28

$(0.78,2.08)$

$(0.64,0.89)$

0.55

$(0.45,0.68)$

$8,346 \quad 356(4.3)$

1.00

$<0.001$

1.00

Triple vessel disease

$$
\begin{aligned}
& \text { No } \\
& \text { Yes }
\end{aligned}
$$

$$
7,800
$$

315 (4.0)

1.00

8,727

336 (3.9)

0.95

$(0.81,1.11)$

0.534

1.00

Left main stem disease

$\begin{array}{llll}\text { No } & 13,370 & 520(3.9) & 1.00 \\ \text { Yes } & 3,157 & 131(4.1) & 1.07\end{array}$

Ejection fraction

$$
\begin{aligned}
& \text { Good (50+ \%) } \\
& \text { Fair (30 to 49\%) }
\end{aligned}
$$

11,869

367 (3.1) 
Table 7 Associations of preoperative factors with stage-3 acute kidney injury (AKI) in the Bristol and Birmingham development sample (complete case data) (Continued)

\begin{tabular}{|c|c|c|c|c|c|c|c|c|}
\hline \multicolumn{9}{|l|}{ Operative priority } \\
\hline Elective & 10,148 & $323(3.2)$ & 1.00 & & & 1.00 & & \\
\hline Urgent & 6,152 & $295(4.8)$ & 1.53 & $(1.30,1.80)$ & & 1.51 & $(1.20,1.88)$ & \\
\hline Emergency/Salvage & 227 & $33(14.5)$ & 5.17 & $(3.52,7.61)$ & $<0.001$ & 1.36 & $(0.75,2.46)$ & 0.002 \\
\hline \multicolumn{9}{|l|}{ Cardiac procedures } \\
\hline CABG only & 10,828 & $294(2.7)$ & 1.00 & & & 1.00 & & \\
\hline Valve only & 2,482 & $102(4.1)$ & 1.54 & $(1.22,1.93)$ & & 1.45 & $(1.05,2.01)$ & \\
\hline CABG+valve & 1,723 & $135(7.8)$ & 3.05 & $(2.47,3.76)$ & & 2.02 & $(1.57,2.61)$ & \\
\hline Other/multiple & 1,494 & $120(8.0)$ & 3.13 & $(2.51,3.90)$ & $<0.001$ & 2.69 & $(2.02,3.57)$ & $<0.001$ \\
\hline
\end{tabular}

CCS, Canadian Cardiovascular Society; NYHA, New York Heart Association; Ml, myocardial infarction; CABG, coronary artery bypass graft; Haemoglobin, g/dL; GFR, mL/min.

AKI score is available as a web-based calculator [24] that is freely available to any researcher or clinician. It can be accessed by any smart phone, tablet or tabletop computer, and can be completed in less than 1 minute. Using a cutoff for the any-stage AKI score of $30 \%$ will select patients for interventional studies with a positive predictive value of $44 \%$ and a negative predictive value of $85 \%$ for AKI. We suggest that this score may be used to identify an enriched patient cohort for inclusion in clinical trials. We did not detect any advantage for our stage-3 AKI score in relation to existing scores. The stage-3 AKI score may have greater utility as a risk adjustment tool for quality assurance, or clinically to assist with informed consent. However it did not demonstrate clear advantages beyond existing score and we have not developed a web-based calculator for this score.

The study has several limitations. First, retrospective analyses of routinely collected data have limitations with respect to data quality, specifically missing data,

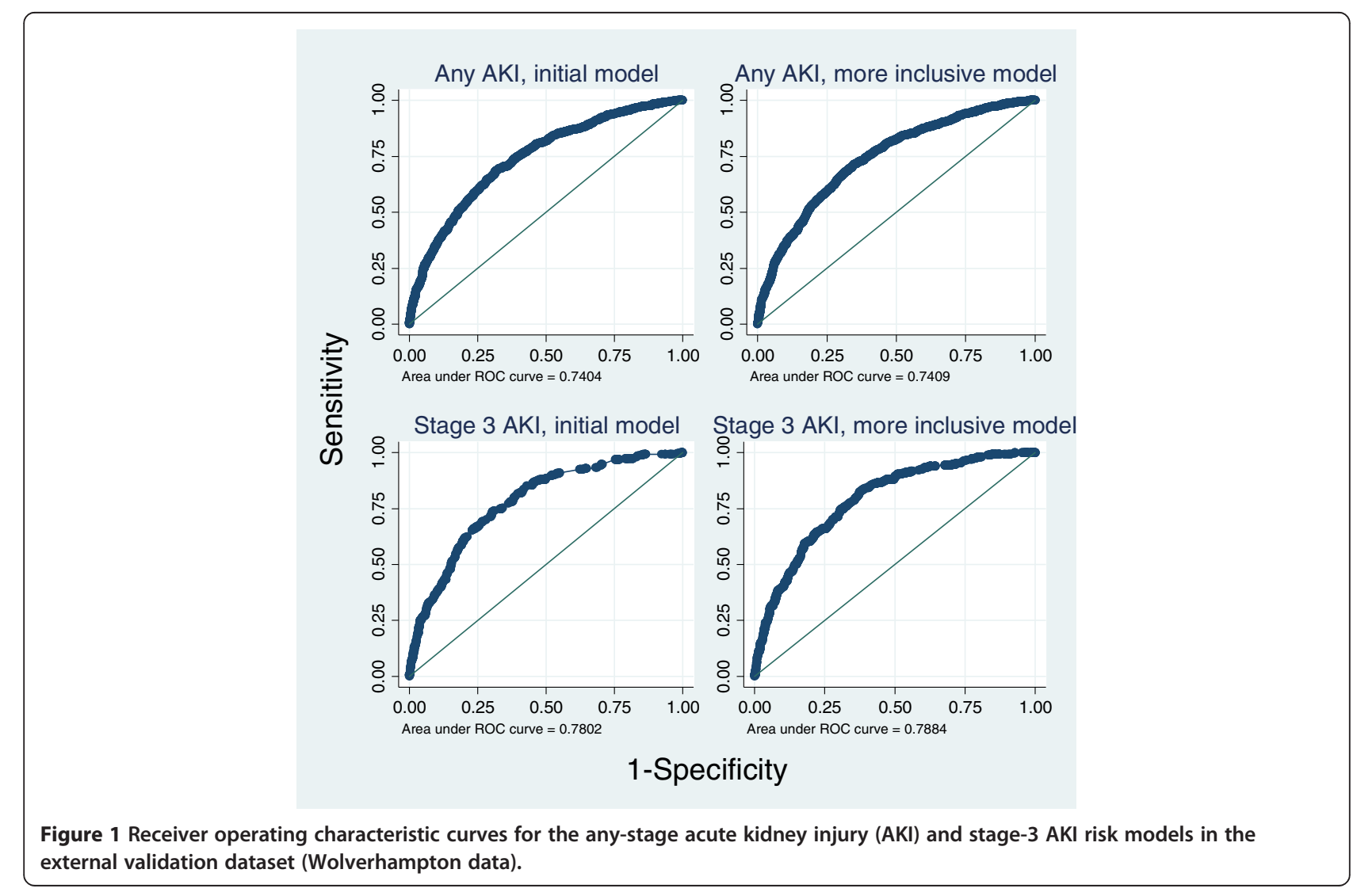


Table 8 Discrimination: area under ROC curves for the different scores and for different procedures (complete case data)

\begin{tabular}{|c|c|c|c|c|}
\hline & \multicolumn{4}{|c|}{ Area under the curve $(95 \% \mathrm{Cl})$} \\
\hline & \multicolumn{2}{|c|}{ Development sample Bristol and Birmingham $n=16,527$} & \multicolumn{2}{|c|}{ Validation sample Wolverhampton $n=4,468$} \\
\hline & Any-stage AKI & Stage-3 AKI & Any-stage AKI & Stage-3 AKI \\
\hline Initial model $(P<0.001)$ & $0.73(0.72,0.74)$ & $0.79(0.77,0.80)$ & $0.74(0.72,0.76)$ & $0.78(0.75,0.80)$ \\
\hline More inclusive model $(P<0.05)$ & $0.74(0.73,0.75)$ & $0.79(0.78,0.81)$ & $0.74(0.72,0.76)$ & $0.79(0.76,0.81)$ \\
\hline \multicolumn{5}{|l|}{ Comparison Scores } \\
\hline Euroscore & $0.66(0.65,0.67)$ & $0.71(0.69,0.73)$ & $0.68(0.66,0.70)$ & $0.73(0.70,0.76)$ \\
\hline Cleveland clinic & $0.65(0.64,0.66)$ & $0.74(0.72,0.76)$ & $0.70(0.69,0.72)$ & $0.78(0.750 .81)$ \\
\hline Metha score & $0.71(0.70,0.72)$ & $0.79(0.77,0.80)$ & $0.74(0.72,0.76)$ & $0.79(0.77,0.82)$ \\
\hline Ng score & $0.70(0.69,0.71)$ & $0.77(0.75,0.79)$ & $0.73(0.71,0.75)$ & $0.79(0.76,0.82)$ \\
\hline \multicolumn{5}{|l|}{ Procedure type } \\
\hline Coronary artery bypass graft only & $0.73(0.72,0.74)$ & $0.78(0.75,0.81)$ & $0.72(0.70,0.74)$ & $0.78(0.73,0.82)$ \\
\hline Valve only & $0.72(0.70,0.74)$ & $0.75(0.70,0.80)$ & $0.73(0.69,0.77)$ & $0.78(0.72,0.84)$ \\
\hline Coronary artery bypass graft + valve & $0.70(0.66,0.72)$ & $0.74(0.70,0.79)$ & $0.71(0.67,0.75)$ & $0.70(0.62,0.76)$ \\
\hline Other/multiple & $0.70(0.67,0.73)$ & $0.69(0.64,0.74)$ & $0.70(0.64,0.76)$ & $0.72(0.62,0.81)$ \\
\hline
\end{tabular}

$P$-values to compare diagnostic utility between our score and existing scores in validation sample for any acute kidney injury (AKI): Euroscore $P<0.001 ;$ Cleveland clinic $P<0.001$; Metha score $P=0.807 ; \mathrm{Ng}$ score $P=0.172$. For stage-3 AKI: Euroscore $P=0.002 ;$ Cleveland clinic $P=0.801 ;$ Metha score $P=0.173 ;$ Ng score $P=0.384$.

misclassification and inconsistent data definitions between individuals and sites. To minimise these we used prospectively collected data from three clinical databases that use common, standardised definitions for clinical risk factors. The data had undergone both internal and external quality checking, had low levels of missing data, and the three sites contributing to the study are listed as among the top for data quality within the UK NACSA programme. Importantly, baseline creatinine values, defined in this study as the preoperative value obtained closest to the date of operation, were present in over $98 \%$ of patients, as would be expected in a cardiac surgery cohort where preoperative bloods are routinely taken in all but the very sickest patients. This

Table 9 Calibration: Hosmer Lemshow tests and results of linear regression analysis of observed versus expected calibration plots

\begin{tabular}{|c|c|c|c|c|c|c|c|c|}
\hline & \multicolumn{4}{|c|}{ Development sample Bristol and Birmingham $n=16,527$} & \multicolumn{4}{|c|}{ Validation sample Wolverhampton $n=4,468$} \\
\hline & \multicolumn{2}{|c|}{ Any-stage AKI } & \multicolumn{2}{|c|}{ Stage-3 AKI } & \multicolumn{2}{|c|}{ Any-stage AKI } & \multicolumn{2}{|c|}{ Stage-3 AKI } \\
\hline Hosmer-Lemeshow $P$-values ${ }^{a}$ & \multicolumn{2}{|l|}{$P$} & \multicolumn{2}{|l|}{$P$} & \multicolumn{2}{|l|}{$P$} & \multicolumn{2}{|l|}{$P$} \\
\hline Initial model $(P<0.001)$ & \multicolumn{2}{|l|}{0.490} & \multicolumn{2}{|l|}{0.001} & \multicolumn{2}{|l|}{0.192} & \multicolumn{2}{|l|}{$<0.001$} \\
\hline More inclusive model $(P<0.05)$ & \multicolumn{2}{|l|}{0.784} & \multicolumn{2}{|l|}{$<0.001$} & \multicolumn{2}{|l|}{0.406} & \multicolumn{2}{|l|}{$<0.001$} \\
\hline Euroscore & \multicolumn{2}{|l|}{$<0.001$} & \multicolumn{2}{|l|}{$<0.001$} & \multicolumn{2}{|l|}{0.333} & \multicolumn{2}{|l|}{$<0.001$} \\
\hline Cleveland clinic & \multicolumn{2}{|l|}{0.112} & \multicolumn{2}{|l|}{$<0.001$} & \multicolumn{2}{|l|}{0.141} & \multicolumn{2}{|l|}{$<0.001$} \\
\hline Metha score & \multicolumn{2}{|l|}{0.009} & \multicolumn{2}{|l|}{$<0.001$} & \multicolumn{2}{|l|}{0.136} & \multicolumn{2}{|l|}{$<0.001$} \\
\hline \multirow[t]{2}{*}{ Ng score } & \multicolumn{2}{|l|}{0.013} & \multicolumn{2}{|l|}{$<0.001$} & \multicolumn{2}{|l|}{0.174} & \multicolumn{2}{|l|}{$<0.001$} \\
\hline & \multicolumn{2}{|c|}{ Any-stage AKI } & Stage- & & Any-st & e AKI & Stage- & \\
\hline Slope and intercept ${ }^{\mathrm{b}}$ & Slope & Intercept & Slope & Intercept & Slope & Intercept & Slope & Intercept \\
\hline Initial model $(P<0.001)$ & 1.045 & -0.007 & 1.094 & -0.002 & 1.152 & -0.009 & 1.529 & 0.009 \\
\hline More inclusive model $(P<0.05)$ & 1.050 & -0.008 & 1.334 & -0.007 & 1.140 & -0.001 & 2.088 & -0.003 \\
\hline Euroscore & 1.050 & -0.009 & 1.145 & -0.004 & 1.152 & -0.038 & 1.789 & -0.016 \\
\hline Cleveland clinic & 1.283 & -0.048 & 1.145 & -0.004 & 1.927 & -0.138 & 3.376 & -0.033 \\
\hline Metha score & 1.144 & -0.029 & 1.513 & -0.013 & 1.348 & -0.040 & 2.199 & -0.010 \\
\hline $\mathrm{Ng}$ score & 1.130 & -0.021 & 1.378 & -0.008 & 1.338 & -0.010 & 2.196 & -0.004 \\
\hline
\end{tabular}

${ }^{\mathrm{a}}$ Higher values indicate better calibration. ${ }^{\mathrm{b}}$ Slope and intercept from linear regression analysis of observed versus expected values analysed by decile, as plotted in Figure 2. The closer the slope is to 1 , and the closer the intercept is to 0 , indicates better calibration. 
A Any stage AKI, Bristol and Birmingham

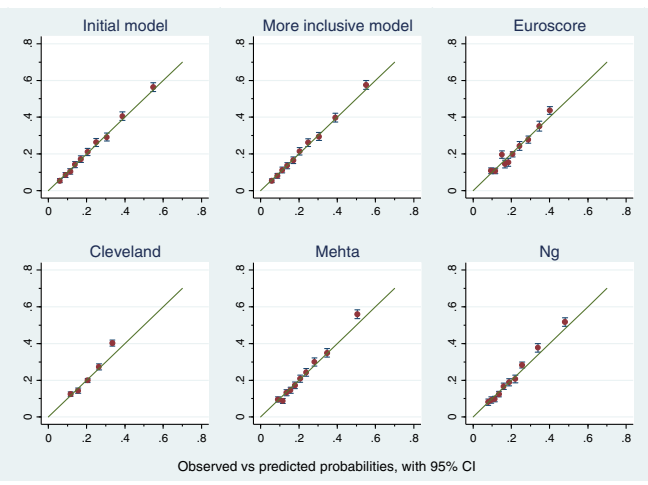

B Any stage AKI, Wolverhampton

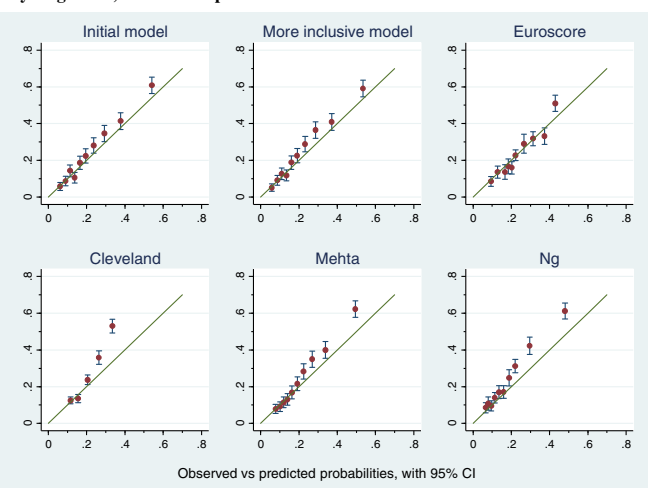

C Stage 3 AKI, Bristol and Birmingham

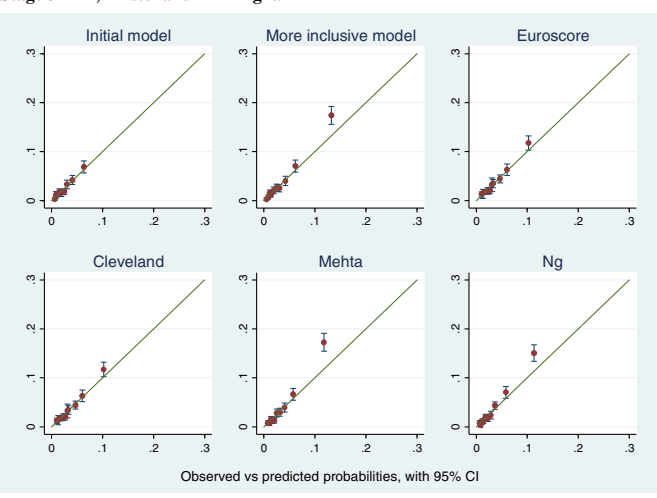

D Stage $3 \mathrm{AKI}$, Wolverhampton

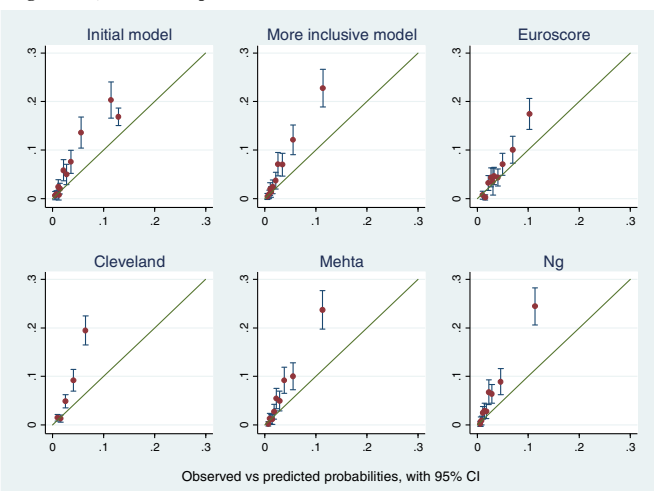

Figure 2 Calibration plots of observed versus expected values for the any-stage acute kidney injury (AKI), stage-3 AKI, Cleveland, Mehta, $\mathrm{Ng}$ score and EuroScore in the development (Bristol, Birmingham) and validation (Wolverhampton) datasets. (A) Any-stage AKI, Bristol and Birmingham. (B) Any-stage AKI, Wolverhampton. (C) Stage-3 AKI, Bristol and Birmingham. (D) Stage-3 AKI, Wolverhampton.

is important; alternate definitions of baseline change the reported frequency of stage-1 AKI [25] - a key consideration in this study. The only variable for which there was a significant proportion of missing data was baseline haemoglobin, and this was restricted to a single centre. To address the limitations posed by missing data we performed our primary analysis in the complete case data. We confirmed the robustness of the models developed in the complete case analysis in imputed data. Model coefficients do not differ substantially in the imputed data, which suggests that the missing data are unlikely to have introduced bias into our model.

Second, unmeasured confounders are an important consideration in any retrospective analysis. For example, the Bristol and Birmingham databases do not routinely record patient race, and this has been found to be an important variable in some risk scores but not others [6]. This may also have affected the estimated GFR (eGFR) calculation, a key component of the score. Equations to calculate eGFR that include race, such as, for example, the modified diet in renal disease equation [26] have greater accuracy.

Third, intra and postoperative events that affect the incidence of AKI also represent confounders [10,27]. However, it was our intention to design a score that will identify patients at risk of AKI preoperatively on the basis that the most effective prevention strategies are likely to be those applied before or at the commencement of surgery rather than after injury (surgery) has occurred. Fourth, the AKI definition used did not incorporate urine output data, as defined in the KDIGO definition, as these data were not recorded. Urine output data are known to significantly alter the estimates in patients with AKI, although whether this improves the prognostic utility of the scores is unclear particularly in cardiac surgery, where perioperative urine output is closely monitored, and oliguria aggressively treated [28]. The use of creatininebased definitions of AKI has other limitations in cardiac surgery. In the validation sample in this study $48 \%$ of patients had undergone coronary angiography within the same hospital admission and 39\% were undergoing urgent inpatient surgery. Many of these patients would have sustained significant renal insults prior to surgery and this may have increased the baseline serum creatinine value. 
Table 10 Model coefficients for final models (complete case data)

\begin{tabular}{|c|c|c|c|c|c|c|}
\hline \multirow{3}{*}{ Age, years } & \multicolumn{3}{|c|}{ Any-stage AKI } & \multicolumn{3}{|l|}{ Stage-3 AKI } \\
\hline & \multirow[t]{2}{*}{ Odds ratio } & \multirow[t]{2}{*}{$95 \% \mathrm{Cl}$} & \multirow[t]{2}{*}{$P$-value } & \multirow[t]{2}{*}{ Odds ratio } & \multirow[t]{2}{*}{$95 \% \mathrm{Cl}$} & \multirow[t]{2}{*}{$P$-value } \\
\hline & & & & & & \\
\hline$<60$ & 1.00 & & & & & \\
\hline 60 to 74 & 1.53 & $(1.35,1.73)$ & & & & \\
\hline$\geq 75$ & 2.19 & $(1.89,2.54)$ & $<0.001$ & & & \\
\hline \multicolumn{7}{|l|}{ Sex } \\
\hline Male & 1.00 & & & 1.00 & & \\
\hline Female & 0.53 & $(0.48,0.59)$ & $<0.001$ & 0.42 & $(0.34,0.51)$ & $<0.001$ \\
\hline \multicolumn{7}{|l|}{ Body mass index } \\
\hline$<20.0$ & 0.80 & $(0.64,0.99)$ & & 0.54 & $(0.35,0.82)$ & \\
\hline 20.0 to 24.9 & 0.80 & $(0.72,0.88)$ & & 0.84 & $(0.68,1.04)$ & \\
\hline 25.0 to 29.9 & 1.00 & & & 1.00 & & \\
\hline 30.0 to 34.9 & 1.75 & $(1.57,1.95)$ & & 1.81 & $(1.44,2.27)$ & \\
\hline $35.0+$ & 2.08 & $(1.76,2.47)$ & $<0.001$ & 2.71 & $(1.94,3.79)$ & $<0.001$ \\
\hline \multicolumn{7}{|l|}{ Smoking status } \\
\hline Never smoked & 1.00 & & & & & \\
\hline Ex smoker & 1.09 & $(1.00,1.20)$ & & & & \\
\hline Current smoker & 1.41 & $(1.22,1.64)$ & $<0.001$ & & & \\
\hline \multicolumn{7}{|l|}{ Dyspnoea } \\
\hline NYHA 1 & 1.00 & & & & & \\
\hline NHYA 2 & 1.05 & $(0.94,1.17)$ & & & & \\
\hline NYHA 3 & 1.29 & $(1.14,1.45)$ & & & & \\
\hline NYHA 4 & 1.44 & $(1.20,1.74)$ & $<0.001$ & & & \\
\hline \multicolumn{7}{|c|}{ Preoperative diabetes } \\
\hline No & 1.00 & & & 1.00 & & \\
\hline Yes & 1.40 & $(1.27,1.55)$ & $<0.001$ & 1.91 & $(1.59,2.31)$ & $<0.001$ \\
\hline \multicolumn{7}{|c|}{ Peripheral vascular disease } \\
\hline No & 1.00 & & & & & \\
\hline Yes & 1.35 & $(1.20,1.52)$ & $<0.001$ & & & \\
\hline \multicolumn{7}{|l|}{ Hypertension } \\
\hline No & 1.00 & & & & & \\
\hline Yes & 1.28 & $(1.17,1.40)$ & $<0.001$ & & & \\
\hline \multicolumn{7}{|l|}{ Haemoglobin } \\
\hline$<10.0$ & 1.68 & $(1.31,2.17)$ & & 2.98 & $(2.05,4.32)$ & \\
\hline 10.0 to 11.9 & 1.71 & $(1.53,1.91)$ & & 2.53 & $(2.08,3.07)$ & \\
\hline $12.0+$ & 1.00 & & $<0.001$ & 1.00 & & $<0.001$ \\
\hline \multicolumn{7}{|c|}{ Glomerular filtration rate } \\
\hline$<30.0$ & 4.58 & $(3.69,5.69)$ & & 9.50 & $(6.89,13.10)$ & \\
\hline 30.0 to 59.9 & 2.25 & $(2.04,2.49)$ & & 2.56 & $(2.10,3.13)$ & \\
\hline 60.0 to 89.9 & 1.00 & & & 1.00 & & \\
\hline $90.0+$ & 0.82 & $(0.72,0.95)$ & $<0.001$ & 0.43 & $(0.30,0.62)$ & $<0.001$ \\
\hline \multicolumn{7}{|c|}{ Critical preoperative event } \\
\hline \multicolumn{7}{|l|}{ No } \\
\hline Yes & & & & 3.35 & $(2.35,4.78)$ & $<0.001$ \\
\hline
\end{tabular}


Table 10 Model coefficients for final models (complete case data) (Continued)

\begin{tabular}{|c|c|c|c|c|c|c|}
\hline \multicolumn{7}{|l|}{ Catheter to surgery } \\
\hline Within $24 \mathrm{~h}$ & 1.22 & $(0.91,1.64)$ & & 1.58 & $(1.03,2.43)$ & \\
\hline$>24$ hrs this admission & 0.78 & $(0.71,0.86)$ & & 0.71 & $(0.59,0.85)$ & \\
\hline$>24$ hrs previous admission & 1.00 & & $<0.001$ & 1.00 & & $<0.001$ \\
\hline \multicolumn{7}{|l|}{ Triple vessel disease } \\
\hline No & 1.00 & & & & & \\
\hline Yes & 1.22 & $(1.10,1.34)$ & $<0.001$ & & & \\
\hline \multicolumn{7}{|l|}{ Ejection fraction } \\
\hline Good (50+ \%) & 1.00 & & & & & \\
\hline Fair (30-49\%) & 1.24 & $(1.14,1.36)$ & & & & \\
\hline Poor $(<30 \%)$ & 1.42 & $(1.20,1.67)$ & $<0.001$ & & & \\
\hline \multicolumn{7}{|l|}{ Operative priority } \\
\hline Elective & 1.00 & & & & & \\
\hline Urgent & 1.43 & $(1.30,1.58)$ & & & & \\
\hline Emergency/salvage & 2.45 & $(1.74,3.43)$ & $<0.001$ & & & \\
\hline \multicolumn{7}{|l|}{ Cardiac procedures } \\
\hline CABG only & 1.00 & & & 1.00 & & \\
\hline Valve only & 1.43 & $(1.24,1.65)$ & & 1.50 & $(1.17,1.92)$ & \\
\hline CABG+valve & 1.89 & $(1.67,2.15)$ & & 2.15 & $(1.72,2.70)$ & \\
\hline Other/multiple & 1.82 & $(1.57,2.11)$ & $<0.001$ & 2.92 & $(2.30,3.72)$ & $<0.001$ \\
\hline Constant & 0.05 & $(0.04,0.06)$ & $<0.001$ & 0.01 & $(0.01,0.02)$ & $<0.001$ \\
\hline
\end{tabular}

Models are the initial models (variables $P<0.001$ in models adjusting for main effects). AKI, acute kidney injury; NYHA, New York Heart Association; CABG, coronary artery bypass graft; Haemoglobin, $\mathrm{g} / \mathrm{dL} ; \mathrm{GFR}, \mathrm{mL} / \mathrm{min}$.

A final limitation is the applicability of our findings to non-UK populations. We used well-defined variables that are routinely collected in UK databases, however, differences in variable definitions that occur between databases and countries may limit the wider utility of the model. This was a limitation of the two North American comparison scores. The Mehta score, in particular was not composed of variables that were routinely collected in the UK data, including $\mathrm{MI}<3$ weeks, and ethnicity. The Mehta score was also developed in a selected population and both American scores excluded patients undergoing surgery without $\mathrm{CPB}$, but were subsequently tested in a UK population that included a significant proportion of off-pump procedures. Conversely variable definitions were largely comparable between the UK and Australian cohorts, and the $\mathrm{Ng}$ score demonstrated good discrimination but poor calibration. This may reflect the non-consensus definition of AKI used in the $\mathrm{Ng}$ score or differences in patient populations and clinical practice between the two countries. These findings highlight the problems of risks scores developed in distinct geographic populations. The UK AKI risk scores described here may suffer from similar limitations, and we conclude that their wider utility requires independent external validation.

\section{Conclusion}

This study has used a large multicentre cohort to develop and validate a risk prediction score for AKI stages 1 to 3 . This is the only published score that predicts less severe AKI, as currently defined by the consensus KDIGO definition. We suggest that this new score core will have clinical utility for risk stratification and facilitate cohort enrichment for clinical trials of novel renoprotective interventions.

\section{Key messages}

- $\mathrm{AKI}$ is a common and severe complication of cardiac surgery that contributes to morbidity, mortality and increased healthcare costs

- Previous trials of renoprotective interventions have been limited by the enrolment of low- or mixed-risk AKI cohorts, and no effective treatment has been identified thus far

- AKI risk scores are an objective and transparent way of identifying cohorts of patients at increased risk of AKI for clinical trials; however, existing scores identify only those patients who develop severe AKI requiring renal replacement therapy 
- This study used data from two large cardiac surgery centres to develop a risk score that identifies all patients at risk of AKI (stages 1 to 3) with high discrimination and good calibration in an external validation dataset from a third centre

- The utility of this score is currently being prospectively validated as a cohort enrichment toll in several ongoing clinical trials

\begin{abstract}
Abbreviations
AKI: acute kidney injury; AKIN: Acute Kidney Injury Network; AUC: area under the curve; BHF: British Heart Foundation; BIPAP: bi-level positive airway pressure; BMI: body mass index; CABG: coronary artery bypass graft surgery; CCS: Canadian Cardiovascular Society; CPAP: continuous positive airway pressure; GFR: glomerular filtration rate; Hb: haemoglobin; HDU: High Dependency Unit; IABP: intra-aortic balloon pump; IQR: Interquartile range; KDIGO: Kidney Disease Improving Global Outcomes; MI: myocardial Infarction; NASCA: National Adult Cardiac Surgery Audit; NHS: National Health Service; NICOR: National Institute Clinical Outcome Research; NYHA: New York Heart Association; PATS: patient analysis and tracking system; RCT: randomised controlled trial; RIFLE: risk injury, failure, loss, end stage; ROC: receiver operating characteristic; RRT: renal replacement therapy.
\end{abstract}

\section{Competing interests}

GJ Murphy has served as a consultant to the AKI divisions of AbbVie and Thrasos Innovation Inc. The remaining authors have no disclosures.

\section{Authors' contributions}

GJM, JAS and W conceived the study and established the funding for the study. GJM, MB and DP were principal investigators for the three institutions and helped design the study. KB, KT and JAS undertook the analyses. KB, GJM and JAS wrote the manuscript. All the authors have reviewed and approved the manuscript.

\section{Acknowledgements}

Thanks to Jessica Harris and Chris Rogers for matching clinical and laboratory data in the Bristol dataset (University of Bristol). Collaborators who contributed data to the study: Birmingham: Mr TR Graham, Mr SJ Rooney, Mr IC Wilson Mr RS Bonser, Mr J Mascaro, Database manager Ms Vivian Barnett; Bristol: Mr M Yeatman, Mr G Asimakopoulos, Mr M Caputo, Mr S Stoica, Mr A Parry, Mr GD Angelini, Mr F Ciulli, Mr R Ascione, Mr J A Hutter, Database manager Mr Alan Davies; Wolverhampton: Mr M Matuszewski, Mr P Yiu, Mr J S Billing, Mr I S Morgan, Mr H Luckraz, Database manager Ms J Sear.

\section{Funding}

This study was funded by a Flexibility and Sustainability Grant from University Hospitals Bristol NHS Foundation Trust, and BHF Grants CH/12/1/ 29419 and RG/13/6/29947, and the Leicester NIHR Cardiovascular Biomedical Research Unit. KB is funded Medical Research Council UK (grant RD1826).

\section{Author details}

${ }^{1}$ School of Social and Community Medicine, University of Bristol, Bristol, UK. ${ }^{2}$ Department of Cardiovascular Sciences, University of Leicester, Leicester NIHR Cardiovascular Biomedical Research Unit, Clinical Sciences Wing, Glenfield General Hospital, Leicester LE3 9QP, UK. ${ }^{3}$ Quality and Outcomes Research Unit, University Hospitals, Birmingham \& School of Experimental Medicine, University of Birmingham, Birmingham, UK. ${ }^{4}$ University Hospitals of Birmingham NHS Foundation Trust, Birmingham, UK.

Received: 9 April 2014 Accepted: 20 October 2014 Published online: 20 November 2014

\section{References}

1. Lassnigg A, Schmidlin D, Mouhieddine M, Bachmann LM, Druml W, Bauer P, Hiesmayr M: Minimal changes of serum creatinine predict prognosis in patients after cardiothoracic surgery: a prospective cohort study. J Am Soc Nephrol 2004, 15:1597-1605.

2. Howell NJ, Freemantle N, Bonser RS, Graham TR, Mascaro J, Rooney SJ, Wilson IC, Pagano D: Subtle changes in renal function are associated with differences in late survival following adult cardiac surgery. Eur $J$ Cardiothorac Surg 2012, 41:e38-e42.

3. Park M, Coca SG, Nigwekar SU, Garg AX, Garwood S, Parikh CR: Prevention and treatment of acute kidney injury in patients undergoing cardiac surgery: a systematic review. Am J Nephrol 2010, 31:408-418.

4. Zacharias M, Mugawar M, Herbison GP, Walker RJ, Hovhannisyan K, Sivalingam $P$, Conlon NP: Interventions for protecting renal function in the perioperative period. Cochrane Database Syst Rev 2013, 9:CD003590.

5. Palevsky PM, Molitoris BA, Okusa MD, Levin A, Waikar SS, Wald R, Chertow GM, Murray PT, Parikh CR, Shaw AD, Go AS, Faubel SG, Kellum JA, Chinchilli VM, Liu KD, Cheung AK, Weisbord SD, Chawla LS, Kaufman JS, Devarajan P, Toto RM, Hsu CY, Greene T, Mehta RL, Stokes JB, Thompson AM, Thompson BT, Westenfelder CS, Tumlin JA, Warnock DG, et al: Design of clinical trials in acute kidney injury: report from an NIDDK workshop on trial methodology. Clin J Am Soc Nephrol 2012, 7:844-850.

6. Huen SC, Parikh CR: Predicting acute kidney injury after cardiac surgery: a systematic review. Ann Thorac Surg 2012, 93:337-347.

7. Thakar CV, Arrigain S, Worley S, Yared J-P, Paganini EP: A clinical score to predict acute renal failure after cardiac surgery. J Am Soc Nephrol 2005, 16:162-168.

8. Mehta RH, Grab JD, O'Brien SM, Glower DD, Haan CK, Gammie JS, Peterson ED, Society of Thoracic Surgeons National Cardiac Database Investigators: Bedside tool for predicting the risk of postoperative dialysis in patients undergoing cardiac surgery. Circulation. 2006, 114:2208-2216.

9. Englberger L, Suri RM, Li Z, Casey ET, Daly RC, Dearani JA, Schaff HV: Validation of clinical scores predicting severe acute kidney injury after cardiac surgery. Am J Kidney Dis 2010, 56:623-631.

10. Ng SY, Sanagou M, Wolfe R, Cochrane A, Smith JA, Reid CM: Prediction of acute kidney injury within 30 days of cardiac surgery. J Thorac CardiovasC Surg 2014, 147:1875-1883.

11. Kidney Disease: Improving Global Outcomes (KDIGO) Acute Kidney Injury Work Group: KDIGO clinical practice guideline for acute kidney injury. Kidney Int 2012, Suppl2:1-138.

12. Mehta RL, Kellum JA, Shah SV, Molitoris BA, Ronco C, Warnock DG, Levin A: Acute Kidney Injury Network: report of an initiative to improve outcomes in acute kidney injury. Crit Care 2007, 11:R31.

13. Bellomo R, Ronco C, Kellum JA, Mehta RL, Palevsky P: Acute renal failure-definition, outcome measures, animal models, fluid therapy and information technology needs: the Second International Consensus Conference of the Acute Dialysis Quality Initiative (ADQI) Group. Crit Care 2004, 8:R204-R212.

14. Bastin AJ, Ostermann M, Slack AJ, Diller GP, Finney SJ, Evans TW: Acute kidney injury after cardiac surgery according to Risk/ Injury/ Failure/ Loss/End-stage, Acute Kidney Injury Network, and Kidney Disease: Improving Global Outcomes classifications. J Crit Care. 2013, 28:389-396.

15. Bridgewater $B$, Kinsman R, Walton P, Keogh B: Demonstrating quality: The Sixth National Adult Cardiac Surgery database report. Dendrite Clinical Systems Ltd: Henley-on-Thames, Oxfordshire, United Kingdom; 2008.

16. Blue Book Online: The Society for Cardiothoracic Surgery in Great Britain \& Ireland. [http://bluebook.scts.org/]

17. National Adult Cardiac Surgery Audit Report 2011-12. [https://www.ucl.ac. uk/nicor/nicor-news-publication/nacsa_report_2011-2012]

18. Nashef SA, Roques F, Hammill BG, Peterson ED, Michel P, Grover FL, Wyse RK, Ferguson TB, EurpSCORE Project Group: Validation of European system for cardiac operative risk evaluation (EuroSCORE) in North American cardiac surgery. Eur J Cardiothorac Surg 2002, 22:101-105.

19. Raghunathan TE, Lepkowski JM, Van Hoewyk J, Solenberger P: A multivariate technique for multiply imputing missing values using a sequence of regression models. Survey Methodology 2009, 27:85-95.

20. Royston P: Multiple imputation of missing value. Stata Journal 2004, 4:227-241.

21. Royston P: Multiple imputation of missing values: update of ice. Stata Journal 2005, 5:527-536.

22. Vergouwe $Y$, Royston P, Moons KGM, Altman DG: Development and validation of a prediction model with missing predictor data: a practical approach. J Clin Epidemiol 2010, 63:205-214.

23. Marshall A, Altman DG, Holder RL, Royston P: Combining estimates of interest in prognostic modelling studies after multiple imputation: current practice and guidelines. BMC Med Res Methodol 2009, 9:57.

24. Leicester cardiac Surgery Research Acute Kidney Injury Risk Score Calculator. [http://www.cardiacsurgeryleicester.com/our-research/acutekidney-injury-risk-score-calculator/] 
25. Siew ED, Matheny ME, Ikizler TA, Lewis JB, Miller RA, Waitman LR, Go AS, Parikh CR, Peterson JF: Commonly used surrogates for baseline renal function affect the classification and prognosis of acute kidney injury. Kidney Int 2010, 77:536-542.

26. Lamb EJ, Stevens PE: Estimating and measuring glomerular filtration rate: methods of measurement and markers for estimation. Curr Opin Nephrol. Hypertens 2014, 23:258-266.

27. Demirjian S, Schold JD, Navia J, Mastracci TM, Paganini EP, Yared JP, Bashour CA: Predictive models for acute kidney injury following cardiac surgery. Am J Kidney Dis 2012, 59:382-389.

28. Md Ralib A, Pickering JW, Shaw GM, Endre ZH: The urine output definition of acute kidney injury is too liberal. Crit Care 2013, 17:R112.

doi:10.1186/s13054-014-0606-x

Cite this article as: Birnie et al.: Predictive models for kidney disease: improving global outcomes (KDIGO) defined acute kidney injury in UK cardiac surgery. Critical Care 2014 18:606.

\section{Submit your next manuscript to BioMed Central and take full advantage of:}

- Convenient online submission

- Thorough peer review

- No space constraints or color figure charges

- Immediate publication on acceptance

- Inclusion in PubMed, CAS, Scopus and Google Scholar

- Research which is freely available for redistribution 